\title{
High-order Higdon-like boundary conditions for exterior transient wave problems ${ }^{\text {II }}$
}

\author{
Vince J. van Joolen ${ }^{1, \ddagger}$, Beny Neta $^{1, *, \dagger}$ and Dan Givoli ${ }^{2, \S}$ \\ ${ }^{1}$ Department of Applied Mathematics, Naval Postgraduate School, 833 Dyer Road, \\ Monterey, CA 93943, U.S.A. \\ ${ }^{2}$ Department of Aerospace Engineering and Asher Center for Space Research, \\ Technion - Israel Institute of Technology, Haifa 32000, Israel
}

\begin{abstract}
SUMMARY
Recently developed non-reflecting boundary conditions are applied for exterior time-dependent wave problems in unbounded domains. The linear time-dependent wave equation, with or without a dispersive term, is considered in an infinite domain. The infinite domain is truncated via an artificial boundary $\mathscr{B}$, and a high-order non-reflecting boundary condition (NRBC) is imposed on $\mathscr{B}$. Then the problem is solved numerically in the finite domain bounded by $\mathscr{B}$. The new boundary scheme is based on a reformulation of the sequence of NRBCs proposed by Higdon. We consider here two reformulations: one that involves high-order derivatives with a special discretization scheme, and another that does not involve any high derivatives beyond second order. The latter formulation is made possible by introducing special auxiliary variables on $\mathscr{B}$. In both formulations the new NRBCs can easily be used up to any desired order. They can be incorporated in a finite element or a finite difference scheme; in the present paper the latter is used. In contrast to previous papers using similar formulations, here the method is applied to a fully exterior two-dimensional problem, with a rectangular boundary. Numerical examples in infinite domains are used to demonstrate the performance and advantages of the new method. In the auxiliary-variable formulation long-time corner instability is observed, that requires special treatment of the corners (not addressed in this paper). No such difficulties arise in the high-derivative formulation. Published in 2005 by John Wiley \& Sons, Ltd.
\end{abstract}

KEY WORDS: $\quad$ waves; high-order; artificial boundary; non-reflecting boundary condition; Higdon; auxiliary variables; finite difference

\footnotetext{
${ }^{*}$ Correspondence to: B. Neta, Department of Applied Mathematics, Naval Postgraduate School, 833 Dyer Road, Monterey, CA 93943, U.S.A.

†E-mail: byneta@gmail.com

‡E-mail: vjvanjoo@nps.navy.mil

${ }^{\S}$ E-mail: givolid@aerodyne.technion.ac.il

IT This article is a U.S. Government work and is in the public domain in the U.S.A.

Contract/grant sponsor: ONR

Contract/grant sponsor: NPS
}

Received 29 January 2004

Revised 18 October 2004

Published in 2005 by John Wiley \& Sons, Ltd.

Accepted 6 December 2004 


\section{INTRODUCTION}

Numerical methods for the solution of wave problems in unbounded domains have been developed since the late 1960s [1]. They have been considered in various fields of application involving wave propagation, such as acoustics, electromagnetics, meteorology, oceanography and geophysics of the solid earth. Among the pioneers were Zienkiewicz and Newton [2] and Lysmer and Kuhlemeyer [3]. Various methods have been proposed, such as the Smith technique [4], boundary integral methods, infinite element methods, absorbing layer methods and nonreflecting boundary condition (NRBC) methods. The present paper concentrates on the latter.

In the method of NRBCs, the infinite domain is truncated via an artificial boundary $\mathscr{B}$, thus dividing the original domain into a finite computational domain $\Omega$ and a residual infinite domain $D$. A special boundary condition is imposed on $\mathscr{B}$, in order to complete the statement of the problem in $\Omega$ (i.e. make the solution in $\Omega$ unique) and, most importantly, to minimize spurious wave reflection that result from $\mathscr{B}$. This boundary condition is called an NRBC, although other names such as absorbing, radiating, open, silent, transmitting, transparent, freespace and pulled-back boundary conditions, are often used too [5]. The problem is then solved numerically in $\Omega$. The setup is illustrated in Figure 1 . In the example shown, $\mathscr{B}$ is the boundary on all four sides of $\Omega$, i.e. $\mathscr{B}=\Gamma_{\mathrm{E}} \cup \Gamma_{\mathrm{N}} \cup \Gamma_{\mathrm{W}} \cup \Gamma_{\mathrm{S}}$.

Naturally, the quality of the numerical solution strongly depends on the properties of the NRBC employed. In the last 25 years or so, much research has been done to develop NRBCs that after discretization lead to a scheme which is stable, accurate, efficient and easy to implement. See References [6-8] for recent reviews on the subject. Of course, it is difficult to find a single NRBC which is ideal in all respects and all cases; this is why the quest for better NRBCs and their associated discretization schemes continues.

Recently, high-order local NRBCs have been introduced. Sequences of increasing-order NRBCs have been available before (e.g. the Bayliss-Turkel conditions [9] constitute such a sequence), but they had been regarded as impractical beyond second or third order from the implementation point of view. Only since the mid 1990s have practical high-order NRBCs been devised.

The first such high-order NRBC was proposed by Collino [10], for two-dimensional timedependent waves in rectangular domains. Its construction requires the solution of the onedimensional wave equation on $\mathscr{B}$. Grote and Keller $[11,12]$ developed an exact NRBC (which can be also regarded as a high-order converging NRBC) for the three-dimensional timedependent wave equation, based on spherical harmonic transformations. They extended this NRBC for the case of elastic waves in Reference [13]. Sofronov [14,15] has independently published a similar scheme in the Russian literature. Hagstrom and Hariharan [16,17] constructed high-order NRBCs for the two- and three-dimensional time-dependent wave equations based on the analytic series representation for the outgoing solutions of these equations. For time-dependent waves in a two-dimensional wave guide, Guddati and Tassoulas [18] devised a high-order NRBC by using rational approximations and recursive continued fractions. Givoli [19] showed how to derive high-order NRBCs for a general class of wave problems, leading to a symmetric finite element formulation. In Reference [20], this methodology was applied to the particular case of time-harmonic waves, using optimally localized Dirichlet-to-Neumann NRBCs.

The presence of wave dispersion makes the time-dependent problem still more difficult as far as NRBC treatment is concerned. Wave dispersion appears in various applications; one important 


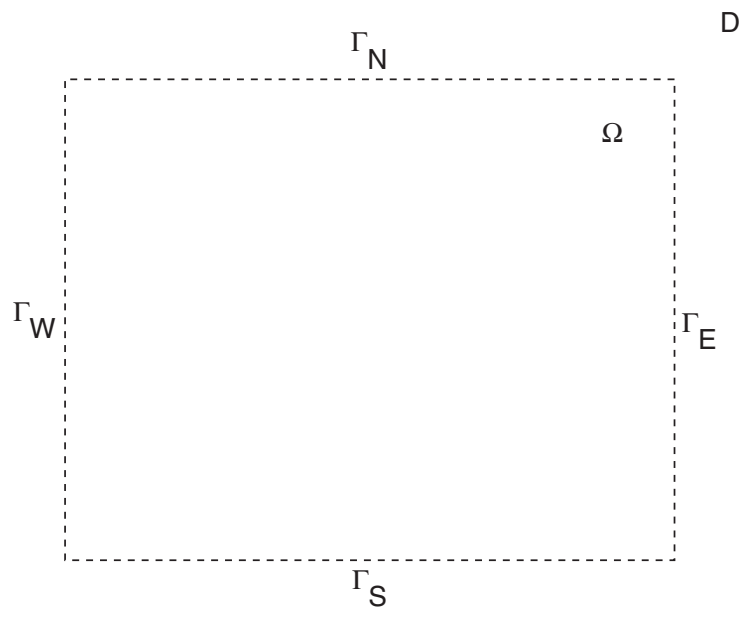

Figure 1. Set-up for the NRBC method: an infinite domain problem.

example is that of meteorological models which take into account the earth rotation [21]. None of the high-order and exact NRBCs mentioned above has been designed to deal with these effects. Several perfectly matched layer (PML) formulations have been proposed for dispersive waves; see, e.g. Reference [22] and references there on a PML scheme for the dispersive shallow water equations.

In References [23,24] we developed high-order NRBC schemes for both dispersive and non-dispersive linear time-dependent waves. The schemes are based on NRBCs which were originally proposed by Higdon [25]. In fact, these NRBCs were developed initially for nondispersive waves [26-30], but Higdon [25] showed that they can be applied in the dispersive case too. The original implementation of the Higdon NRBCs was limited to low orders. Givoli and Neta [23] proposed a new implementation method that allows the use of high-order discretized Higdon NRBCs. However, this method differs from the original Higdon formulation only on the discrete level, not on the continuous level; thus, like the original Higdon scheme, it involves high normal and temporal derivatives, of increasing order. This has several disadvantages which we allude to in the next section. In addition, the computational effort required by the scheme devised in Reference [23] grows exponentially with the order of the NRBC. In a follow-on paper, Givoli and Neta [24] reformulated the Higdon NRBCs on the continuous level in a completely new way. This formulation does not involve any high derivatives. This is made possible by introducing special auxiliary variables on $\mathscr{B}$. The new construction allows the easy use of a Higdon-type NRBC of any desired order, and can be incorporated in a finite element (FE) or a finite difference (FD) scheme. Givoli and Neta [24] and Givoli et al. [31] used, respectively, FDs and FEs to discretize both the partial differential equation in $\Omega$ and the NRBC on $\mathscr{B}$. The computational effort required by the scheme grows only linearly with the order. In this paper we address both the high-derivative formulation and the auxiliary-variable formulation. We discuss the relative advantages of these formulations, and use both of them in the numerical examples presented.

The development in $[23,24,31]$ was limited to the configuration of a wave guide, where $\mathscr{B}$ is a single plane boundary (say $\Gamma_{\mathrm{E}}$ in Figure 1). In the present paper, we extend the results of 
Givoli and Neta $[23,24]$ to the case that two adjacent sides or all four sides of the rectangular domain $\Omega$ require NRBCs. In this case $\mathscr{B}$ has corners which may potentially lead to numerical instabilities. As Higdon [25,28] showed, the high-derivative formulation mentioned above (a high-order implementation of which is presented in Reference [23]) is stable under reasonable conditions, and there is no need to treat the corners specially. Our numerical experiments confirm this fact. Ramahi [32] has reported instabilities for $J=4$ when Higdon's NRBC is combined with the Yee scheme, but they seem to occur only for very special initial conditions. In contrast, in the auxiliary-variable formulation a long-time instability may arise unless special corner conditions are imposed. The papers by Collino [10], Bamberger et al. [33], and very recently Vacus [34] and Hagstrom and Warburton [35], address this problem and devise corner conditions which guarantee a stable solution. In the present paper no special treatment is applied to the corners; indeed a long-time instability is observed for the auxiliary-variable formulation as expected. Special corners conditions may be devised similarly to the treatment in Reference [35]; however, this issue is beyond the scope of this paper.

Following is the outline of the rest of this paper. In Section 2, we state the problem under investigation. We also present the Higdon NRBCs and recall their properties. In Section 3, we present the two new formulations of the Higdon NRBCs and discuss the relative advantages of each. In Section 4, we demonstrate the performance of both formulations of the new method via some numerical examples involving an infinite domain. We conclude with some remarks in Section 5.

\section{STATEMENT OF THE PROBLEM}

We consider wave propagation in a two-dimensional infinite domain as described in Figure 1. Consider the linear inhomogeneous Klein-Gordon equation,

$$
\frac{\partial^{2} \eta}{\partial t^{2}}-C_{0}^{2}\left(\frac{\partial^{2} \eta}{\partial x^{2}}+\frac{\partial^{2} \eta}{\partial y^{2}}\right)+f^{2} \eta=S
$$

In (1), $\eta$ is the unknown wave field, $C_{0}$ is the given reference wave speed, $f$ is the given dispersion parameter, and $S$ is a given wave source function. The $C_{0}$ and $f$ are allowed to be functions of location in a finite region, outside of which they are constant. The wave source $S$ is a function of location and time, but it is assumed to have a local support.

It can be shown that the linearized shallow water equations about a zero mean and with a flat bottom reduce to (1), where $\eta$ is the water elevation above the reference level [21]. In the geophysical context, $f$ is called the Coriolis parameter and is related to the angular velocity of the earth.

The initial conditions

$$
\eta(x, y, 0)=\eta_{0}, \quad \frac{\partial \eta(x, y, 0)}{\partial t}=\eta_{0}^{\prime}
$$

are given at time $t=0$ in the entire domain. We assume that the functions $\eta_{0}$ and $\eta_{0}^{\prime}$ have a local support.

We now truncate the infinite domain by introducing an artificial boundary $\mathscr{B}=\Gamma_{\mathrm{E}} \cup \Gamma_{\mathrm{N}} \cup$ $\Gamma_{\mathrm{W}} \cup \Gamma_{\mathrm{S}}$; see Figure 1. This boundary divides the original infinite domain into two subdomains: 
an exterior domain $D$, and a finite computational domain $\Omega$ which is bounded by $\Gamma_{\mathrm{W}}, \Gamma_{\mathrm{N}}, \Gamma_{\mathrm{S}}$ and $\Gamma_{\mathrm{E}}$. We choose the location of the four sides such that the entire support of $S, \eta_{0}, \eta_{0}^{\prime}$, as well as the region of non-uniformity of $C_{0}$ and $f$, are all contained inside $\Omega$. Thus, on $\mathscr{B}$ and in $D$, the homogeneous counterpart of (1) holds, i.e.

$$
\frac{\partial^{2} \eta}{\partial t^{2}}-C_{0}^{2}\left(\frac{\partial^{2} \eta}{\partial x^{2}}+\frac{\partial^{2} \eta}{\partial y^{2}}\right)+f^{2} \eta=0
$$

with constant coefficients $C_{0}^{2}$ and $f^{2}$, and the medium is initially at rest.

To obtain a well-posed problem in the finite domain $\Omega$ we need to impose a boundary condition on $\mathscr{B}$. We do this by using a NRBC on $\mathscr{B}$, with the aim of preventing the generation of significant spurious reflection of waves. We shall use two reformulations of the Higdon $N R B C$ [25]. The first one differs from the original Higdon formulation only on the discrete level, and as such will involve high-order normal and temporal derivatives. The second one will only require second-order tangential and temporal derivatives. The Higdon NRBC of order $J$ is

$$
H_{J}:\left[\prod_{j=1}^{J}\left(\frac{\partial}{\partial t}+C_{j} \frac{\partial}{\partial v}\right)\right] \eta=0 \quad \text { on } \mathscr{B}
$$

Here, $\partial / \partial v$ is the normal derivative and the $C_{j}$ are constant parameters which have to be chosen and which signify phase speeds in the direction normal to the boundary.

One important property of the Higdon conditions is the following. It is easy to show [25] that when a plane wave which has phase velocity $C_{n}$ in the direction normal to the boundary impinges on the boundary where the NRBC $H_{J}$ is imposed, the resulting reflection coefficient is

$$
R=\prod_{j=1}^{J}\left|\frac{C_{j}-C_{n}}{C_{j}+C_{n}}\right|
$$

It is thus clear that if $C_{j}=C_{n}$ for any one of the $j$ 's then $R=0$, namely there is no reflection and the NRBC is exact. More importantly, $R$ in (5) is a product of $J$ factors, each of which is smaller than 1. This implies that the reflection coefficient becomes smaller as the order $J$ increases regardless of the choice made for the parameters $C_{j}$. Of course, a good choice for the $C_{j}$ would lead to better accuracy with a lower order $J$, but even if the 'wrong' $C_{j}$ 's are taken (say, if one makes the simplest choice $C_{j}=C_{0}$ for $j=1, \ldots, J$ ), one is still guaranteed to reduce the spurious reflection as the order $J$ increases. This has been confirmed by numerical experimentation. The main advantages of the Higdon conditions are listed in Reference [24].

It can be shown that for certain choices of the parameters, the Higdon NRBCs are equivalent to NRBCs that are derived from rational approximation of the dispersion relation (the EngquistMajda conditions being the most well-known example). This has been proved by Higdon [25] and in earlier papers. Thus, the Higdon NRBCs can be viewed as generalization of rationalapproximation NRBCs. See also the recent paper by Ditkowski and Gottlieb [36] on this subject.

We note that the first-order condition $H_{1}$ is a Sommerfeld-like boundary condition. If we set $C_{1}=C_{0}$ we get the classical Sommerfeld-like NRBC. A lot of work in the meteorological literature is based on using $H_{1}$ with a specially chosen $C_{1}$. Pearson [37] used a special but 
constant value of $C_{1}$, while in the scheme devised by Orlanski [38] and in later improved schemes [39-42] the $C_{1}$ changes dynamically and locally in each time-step based on the solution from the previous time-step. Some of the limited-area weather prediction codes used today are based on such schemes, e.g. COAMPS [43]. See also the recent papers [44-46] where several such adaptive $H_{1}$ schemes are compared.

Difficulties associated with the original formulation of the Higdon NRBCs (see Reference [25]) are as follows:

- The discrete Higdon conditions were developed in the literature up to third order only, because of their algebraic complexity which increases rapidly with the order.

- The original $J$ th-order Higdon NRBC involves high normal and temporal derivatives, up to order $J$. In fact, it has the form

$$
\sum_{j=0}^{J} \gamma_{j} \frac{\partial^{j}}{\partial v^{j}} \frac{\partial^{J-j}}{\partial t^{J-j}} \eta=0
$$

which is obtained by expanding (4). The high derivatives pose obvious disadvantages. High normal derivatives are problematic; when FEs are used only the $J=1$ condition is compatible with standard (low-order $C^{0}$ ) elements, whereas when FDs are used, the discrete stencil must be a non-standard high-order one, penetrating deeply into the computational domain away from the artificial boundary. High time derivatives are also disadvantageous in that they require the use of high-order time discretization and the storage of the solution history.

The new formulations presented in the next section overcome all these difficulties. Both reformulations allow the easy use of Higdon NRBCs up to an arbitrarily high order. The scheme is coded once and for all for any order; the order of the scheme is simply an input parameter. Of course, since the value of $J$ affects the amount of storage needed, the statement above assumes that the storage issue is generically addressed, e.g. by the use of a dynamic storage scheme.

\section{HIGH-ORDER NRBCS}

\subsection{Reformulation using high derivatives on a discrete level}

The Higdon condition $H_{J}$ is a product of $J$ operators of the form $(\partial / \partial t)+C_{j}(\partial / \partial v)$. To fix ideas, in this section we consider the east boundary $\Gamma_{\mathrm{E}}$ where $\partial / \partial v=\partial / \partial x$. The ideas are easily carried over to the three other boundaries as well. We consider the following FD approximations:

$$
\frac{\partial}{\partial t} \simeq \frac{I-S_{t}^{-}}{\Delta t}, \quad \frac{\partial}{\partial x} \simeq \frac{I-S_{x}^{-}}{\Delta x}
$$

In (7), $\Delta t$ and $\Delta x$ are, respectively, the time-step size and grid spacing in the $x$ direction, $I$ is the identity operator, and $S_{t}^{-}$and $S_{x}^{-}$are shift operators defined by

$$
S_{t}^{-} \eta_{p q}^{n}=\eta_{p q}^{n-1}, \quad S_{x}^{-} \eta_{p q}^{n}=\eta_{p-1, q}^{n}
$$


Here and elsewhere, $\eta_{p q}^{n}$ is the FD approximation of $\eta(x, y, t)$ at grid point $\left(x_{p}, y_{q}\right)$ and at time $t_{n}$. We use (7) in (4) to obtain:

$$
\left[\prod_{j=1}^{J}\left(\frac{I-S_{t}^{-}}{\Delta t}+C_{j} \frac{I-S_{x}^{-}}{\Delta x}\right)\right] \eta_{E q}^{n}=0
$$

Here, the index $E$ correspond to a grid point on the boundary $\Gamma_{\mathrm{E}}$. Higdon has solved this difference equation (and also a slightly more involved equation that is based on time- and space-averaging approximations for $\partial / \partial x$ and $\partial / \partial t$ ) for $J \leqslant 3$ to obtain an explicit formula for $\eta_{E q}^{n}$. This formula is used to find the current values on the boundary $\Gamma_{\mathrm{E}}$ after the solution in the interior points and on the other boundaries has been updated. The formula for $J=2$ is found in Reference [30], and the one for $J=3$ appears in the appendix of Reference [29]. The algebraic complexity of these formulas increases rapidly with the order $J$. Now we show how to implement the Higdon NRBCs to any order using a simple algorithm. To this end, we first multiply (9) by $\Delta t$ and rearrange to obtain

$$
Z \equiv\left[\prod_{j=1}^{J}\left(a_{j} I+d_{j} S_{t}^{-}+e_{j} S_{x}^{-}\right)\right] \eta_{E q}^{n}=0
$$

where

$$
\begin{aligned}
& a_{j}=1+\frac{C_{j} \Delta t}{\Delta x} \\
& d_{j}=-1 \\
& e_{j}=-\frac{C_{j} \Delta t}{\Delta x}
\end{aligned}
$$

The coefficient $d_{j}$ actually does not depend on $j$, but we keep this notation to allow easy extensions to the scheme. Now, $Z$ in (10) can be written as a sum of $3^{J}$ terms, each one is an operator acting on $\eta_{E q}^{n}$, namely

$$
Z \equiv \sum_{m=0}^{3^{J}-1} A_{m} P_{m} \eta_{E q}^{n}=0
$$

Here $A_{m}$ is a coefficient depending on the $a_{j}, d_{j}$ and $e_{j}$, and $P_{m}$ is an operator involving products of $I, S_{t}^{-}$and $S_{x}^{-}$. All the terms in the sum in (14) are computable at the current time step $n$, except the one which involves only the identity operator and no shift operators. If we let this term correspond to $m=0$, then $P_{0}=I$ and

$$
A_{0}=\prod_{j=1}^{J} a_{j}
$$

Thus we get from (14)

$$
Z \equiv A_{0} \eta_{E q}^{n}+Z^{*}=0
$$


Box 1. Algorithm for implementing the Higdon NRBC of order $J$ on the discrete level (first formulation).

- Start with $Z^{*}=0$. Calculate $A_{0}=\prod_{j=1}^{J} a_{j}$.

- Loop over the integers $m=1, \ldots, 3^{J}-1$.

o For a given $m$, transform $m$ into a number $r$ in base 3, consisting of the digits 0,1 and 2 only. The length of $r$ will be at most $J$ digits. Store the $J$ digits of $r$ in the vector $D_{r}(j), j=1, \ldots, J$.

Example: Suppose that $J=6$ and $m=227$. Since 227 in base 3 is $r=22102$, we will get $D_{r}=\left\{\begin{array}{llllll}0 & 2 & 2 & 1 & 0 & 2\end{array}\right\}$.

$\circ$ Use $D_{r}$ to calculate the coefficient $A_{m}$. To this end, start with $A_{m}=1$, loop over $j=1, \ldots, J$, and for each $j$ multiply $A_{m}$ by the factor $a_{j}$ (if $D_{r}(j)=0$ ) or $d_{j}$ (if $\left.D_{r}(j)=1\right)$ or $e_{j}$ (if $D_{r}(j)=2$ ).

Example: For $J=6$ and $m=227$, we have received the vector $D_{r}$ above. Then $A_{227}=a_{1} e_{2} e_{3} d_{4} a_{5} e_{6}$.

○ Use $D_{r}$ to calculate the operator action $P_{m} \eta_{E q}^{n}$. To this end, start with $\hat{n}=n$ and $\hat{i}=E$, loop over $j=1, \ldots, J$, and for each $j$ subtract 1 from $\hat{n}$ (if $D_{r}(j)=1$ ) or subtract 1 from $\hat{i}$ (if $D_{r}(j)=2$ ) or do nothing (if $D_{r}(j)=0$ ). After the loop ends we have $P_{m} \eta_{E q}^{n}=\eta_{\hat{i} q}^{\hat{n}}$.

Example: For the case $J=6$ and $m=227$ considered above, we get $\hat{n}=n-1$ (because the digit ' 1 ' appears only once in $D_{r}$ ), and $\hat{i}=E-3$ (because the digit '2' appears three times in $D_{r}$ ). Hence $P_{227} \eta_{E q}^{n}=\eta_{E-3, q}^{n-1}$.

○ Update: $Z^{*} \leftarrow Z^{*}+A_{m} \eta_{\hat{i} q}^{\hat{n}}$.

- Next $m$.

- $\eta_{E q}^{n}=-Z^{*} / A_{0}$.

where

$$
Z^{*}=\sum_{m=1}^{3^{J}-1} A_{m} P_{m} \eta_{E q}^{n}
$$

From (16) we get

$$
\eta_{E q}^{n}=-Z^{*} / A_{0}
$$

which is the desired value of $\eta$ on the boundary $\Gamma_{\mathrm{E}}$.

The problem now reduces to calculating $Z^{*}$ given by (17). We do this using the algorithm described in Box 1. The basic idea is to calculate the coefficients $A_{m}$ and the operator actions $P_{m} \eta_{E q}^{n}$ term by term. This is done systematically by transforming the integer counter $m$ to a number in base 3 with $J$ digits. The $A_{m}$ and $P_{m}$ are not simple functions of the decimal representation of the number $m$, but they are simple functions of the digits of the base- 3 representation of $m$.

Note that we need to store $\eta_{\hat{i} q}^{\hat{n}}$ values for $\hat{i}=E, E-1, \ldots, E-J$ and $\hat{n}=n, n-1, \ldots, n-J$. In other words, we have to store the history of the values of $\eta$ for a layer of thickness $J+1$ 
points near the boundary $\Gamma_{\mathrm{E}}$ and for $J+1$ time levels (including the current one). If there are $N_{y}$ grid points in the $y$ direction, then the amount of storage needed in a simple storage scheme is $(J+1)^{2} N_{y}$. However, one can save in storage by exploiting the fact that not all values $\eta_{\hat{i} q}^{\hat{n}}$ are needed, but only those for which $(E-\hat{i})+(n-\hat{n}) \leqslant J$. This is clear from (6) and also from (10). For example, the solution at time $t_{n-J}$ should be stored only for points on the boundary $\Gamma_{\mathrm{E}}$ itself.

This formulation of the Higdon NRBCs requires penetration into the domain (because of the high normal derivative) and also requires time-history. Thus the storage requirement can be high. Also, in order to work properly the method requires a 'buffer zone' of zero initial conditions, of width $J$. In addition it is not easy to extend the algorithm to FE formulations and to unstructured meshes. On the other hand, as Higdon [25, 28] showed and our experiments confirm, it is stable at the corners.

\subsection{Reformulation using auxiliary variables}

We first replace the Higdon condition (4) by the equivalent condition

$$
H_{J}:\left[\prod_{j=1}^{J}\left(\frac{\partial}{\partial v}+\frac{1}{C_{j}} \frac{\partial}{\partial t}\right)\right] \eta=0 \quad \text { on } \mathscr{B}
$$

Again, to fix ideas we consider $\Gamma_{\mathrm{E}}$ where $\partial / \partial v=\partial / \partial x$. Now we introduce the auxiliary functions $\phi_{1}, \ldots, \phi_{J-1}$, which are defined on $\mathscr{B}$ as well as in the exterior domain $D$. Eventually we shall use these functions only on $\mathscr{B}$, but the derivation requires that they be defined in $D$ as well, or at least in a non-vanishing region adjacent to $\mathscr{B}$. The functions $\phi_{j}$ are defined via the relations

$$
\begin{aligned}
\left(\frac{\partial}{\partial x}+\frac{1}{C_{1}} \frac{\partial}{\partial t}\right) \eta & =\phi_{1} \\
\left(\frac{\partial}{\partial x}+\frac{1}{C_{2}} \frac{\partial}{\partial t}\right) \phi_{1} & =\phi_{2} \\
\vdots & \\
\left(\frac{\partial}{\partial x}+\frac{1}{C_{J}} \frac{\partial}{\partial t}\right) \phi_{J-1} & =0
\end{aligned}
$$

By definition, these relations hold in $D$, and also on $\mathscr{B}$. It is easy to see that (20)-(22), when imposed as boundary conditions on $\mathscr{B}$, are equivalent to the single boundary condition (19). If we also define

$$
\phi_{0} \equiv \eta, \quad \phi_{J} \equiv 0
$$

then we can write (20)-(22) concisely as

$$
\left(\frac{\partial}{\partial x}+\frac{1}{C_{j}} \frac{\partial}{\partial t}\right) \phi_{j-1}=\phi_{j}, \quad j=1, \ldots, J
$$


This set of conditions involves only first-order derivatives. However, due to the appearance of the $x$ derivative in (24), one cannot discretize the $\phi_{j}$ on the boundary alone. Therefore we shall manipulate (24) in order to get rid of the $x$ derivative.

The function $\eta$ satisfies the wave equation (3) in $D$. The function $\phi_{1}$ is obtained by applying a linear constant coefficient operator to $\eta$, as in (20); hence it is clear that $\phi_{1}$ also satisfies the same equation in $D$. Similarly, we deduce that each of the functions $\phi_{j}$ satisfies a wave equation like (3), namely,

$$
\frac{\partial^{2} \phi_{j}}{\partial x^{2}}+\frac{\partial^{2} \phi_{j}}{\partial y^{2}}-\frac{1}{C_{0}^{2}} \frac{\partial^{2} \phi_{j}}{\partial t^{2}}-\frac{f^{2}}{C_{0}^{2}} \phi_{j}=0
$$

Here we need the assumption that $C_{0}$ and $f$ do not depend on $x$ or on $t$. Now, we make use of the following identity:

$$
\frac{\partial^{2} \phi_{j}}{\partial x^{2}}=\left(\frac{\partial}{\partial x}-\frac{1}{C_{j+1}} \frac{\partial}{\partial t}\right)\left(\frac{\partial}{\partial x}+\frac{1}{C_{j+1}} \frac{\partial}{\partial t}\right) \phi_{j}+\frac{1}{C_{j+1}^{2}} \frac{\partial^{2} \phi_{j}}{\partial t^{2}}
$$

Substituting (26) in (25) and replacing $j$ with $j-1$ everywhere yields, for $j=1, \ldots, J$,

$$
\left(\frac{\partial}{\partial x}-\frac{1}{C_{j}} \frac{\partial}{\partial t}\right)\left(\frac{\partial}{\partial x}+\frac{1}{C_{j}} \frac{\partial}{\partial t}\right) \phi_{j-1}+\left(\frac{1}{C_{j}^{2}}-\frac{1}{C_{0}^{2}}\right) \frac{\partial^{2} \phi_{j-1}}{\partial t^{2}}+\frac{\partial^{2} \phi_{j-1}}{\partial y^{2}}-\frac{f^{2}}{C_{0}^{2}} \phi_{j-1}=0
$$

From this and (24) we get, for $j=1, \ldots, J$,

$$
\left(\frac{\partial}{\partial x}-\frac{1}{C_{j}} \frac{\partial}{\partial t}\right) \phi_{j}+\left(\frac{1}{C_{j}^{2}}-\frac{1}{C_{0}^{2}}\right) \frac{\partial^{2} \phi_{j-1}}{\partial t^{2}}+\frac{\partial^{2} \phi_{j-1}}{\partial y^{2}}-\frac{f^{2}}{C_{0}^{2}} \phi_{j-1}=0
$$

On the other hand, (24) can also be written as

$$
\left(\frac{\partial}{\partial x}+\frac{1}{C_{j+1}} \frac{\partial}{\partial t}\right) \phi_{j}=\phi_{j+1}, \quad j=0, \ldots, J-1
$$

We subtract (28) from (29) to finally obtain, for $j=1, \ldots, J-1$,

$$
\left(\frac{1}{C_{j}}+\frac{1}{C_{j+1}}\right) \frac{\partial \phi_{j}}{\partial t}=\phi_{j+1}+\left(\frac{1}{C_{j}^{2}}-\frac{1}{C_{0}^{2}}\right) \frac{\partial^{2} \phi_{j-1}}{\partial t^{2}}+\frac{\partial^{2} \phi_{j-1}}{\partial y^{2}}-\frac{f^{2}}{C_{0}^{2}} \phi_{j-1}
$$

As desired, the new boundary condition (30) does not involve normal derivatives. In addition, there are no high tangential and temporal derivatives in (30) beyond second order.

Rewriting (20), (30) and (23), we can summarize the new formulation of the $J$ th-order $\mathrm{NRBC}$ on $\Gamma_{\mathrm{E}}$ as follows:

$$
\begin{aligned}
& \beta_{0} \frac{\partial \phi_{0}}{\partial t}+\frac{\partial \phi_{0}}{\partial x}=\phi_{1} \\
& \beta_{j} \frac{\partial \phi_{j}}{\partial t}-\alpha_{j} \frac{\partial^{2} \phi_{j-1}}{\partial t^{2}}-\frac{\partial^{2} \phi_{j-1}}{\partial y^{2}}+\lambda \phi_{j-1}=\phi_{j+1}, \quad j=1, \ldots, J-1
\end{aligned}
$$




$$
\begin{aligned}
& \alpha_{j}=\frac{1}{C_{j}^{2}}-\frac{1}{C_{0}^{2}}, \quad \beta_{0}=\frac{1}{C_{1}}, \quad \beta_{j}=\frac{1}{C_{j}}+\frac{1}{C_{j+1}}, \quad \lambda=\frac{f^{2}}{C_{0}^{2}} \\
& \phi_{0} \equiv \eta, \quad \phi_{J} \equiv 0
\end{aligned}
$$

It should be emphasized that (31)-(34) are equivalent to the Higdon conditions in their original form, (4). No further approximation is added beyond the one entailed in the original Higdon NRBCs, except that it has been assumed that $C_{0}$ is constant in the far field. The crucial reflection property (5) of the latter applies to (31)-(34) as well.

Analogous equations are easily obtained for $\Gamma_{\mathrm{N}}, \Gamma_{\mathrm{W}}$ and $\Gamma_{\mathrm{S}}$. For example, on $\Gamma_{\mathrm{N}}$ (31)-(34) hold with $x$ and $y$ exchanged in (31) and (32). Equations (31)-(34) can be discretized using FDs or FEs. See Reference [24,31] for more details in the context of wave guides.

It should be remarked that in the case of FE discretization (which is not considered here), standard $C^{0}$ shape functions can be used for all the variables $\phi_{j}$, owing to the fact that at most second-order derivatives appear in (31)-(34). Moreover, as the results in Reference [31] show, a stable solution is obtained with simple equal-order interpolation (e.g. bilinear shape functions) for all the variables. Of course, no standard formulation would be possible if (4) were to be discretized directly by FEs, due to the appearance of high derivatives.

Note the appearance of the second-order time derivative of the previous solution in Equation (32). This term may potentially pose stability difficulties, although this has not been thoroughly checked or analysed as yet. Note also that with the choice $C_{j}=C_{0}$ for all $j$ this term drops out.

This formulation is more general than the previous one and does not require penetration into the domain and the keeping of time-history. It also does not require a 'buffer zone' of zero initial conditions. On the other hand, it is potentially less stable (not every combination of $C_{j}$ in every order gives stability) and it may require special attention at the corners.

\section{NUMERICAL EXPERIMENTS}

In this section, we report on several examples showing the benefit of NRBCs using both Higdon reformulations. Table I giving the CPU overhead required as a function of the order $J$ of the NRBC, for a typical example. The first column is the order $J$, the second column shows the time for the auxiliary-variable formulation, and the last column shows the timing for the high-derivative formulation. Notice that the overhead in the latter case is so high that we did not show the cost for $J>11$. The CPU time is given on a Pentium IV, $1.5 \mathrm{GHz}$ with $256 \mathrm{MB}$ RAM PC. For small $J$ values the boundary treatment is negligible compared to the overall effort, hence the CPU times shown are almost constant. For larger $J$ 's, the time for the auxiliary-variable formulation increases linearly with $J$, while that for the high-derivative formulation increases exponentially with $J$, as expected from the theory.

We refer the reader to Givoli and Neta [23] where we have applied the Higdon high-order NRBC to a simple wave guide problem whose exact solution is synthesized. We have shown that automatic choice of $C_{j}$ according to the scheme given in Reference [23] would allow the 
Table I. Overhead cost.

\begin{tabular}{lcc}
\hline & \multicolumn{2}{c}{ CPU (s) } \\
\cline { 2 - 3 } Order & Aux. var. formul. & High deriv. formul. \\
\hline 1 & 0.016 & 0.016 \\
2 & 0.016 & 0.031 \\
3 & 0.016 & 0.016 \\
4 & 0.016 & 0.016 \\
5 & 0.015 & 0.016 \\
6 & 0.016 & 0.093 \\
7 & 0.015 & 0.172 \\
8 & 0.016 & 0.672 \\
9 & 0.047 & 4.42 \\
10 & 0.016 & 35.1 \\
11 & 0.046 & 355 \\
12 & 0.032 & - \\
13 & 0.062 & - \\
14 & 0.047 & - \\
15 & 0.063 & - \\
16 & 0.078 & - \\
17 & 0.078 & - \\
18 & 0.094 & - \\
19 & 0.093 & \\
20 & 0.110 & \\
\hline
\end{tabular}

user to get higher accuracy with $J=5$ than the case with $C_{j}=1$ for $1 \leqslant j \leqslant 7$. In the examples presented in this paper we choose uniform $C_{j}$ (i.e. $C_{j}=C_{0}$ ) since even with such a choice satisfactory accuracy is obtained for sufficiently high $J$. This is due to the guaranteed reduction in the reflection coefficient for increasing $J$ 's (see discussion in Section 2). This choice is the simplest choice that one can make in the lack of any other information of the wave content of the unknown solution.

In Sections 4.1-4.3 we use the high-derivative formulation, while in Section 4.4 we employ the auxiliary-variable formulation. In both cases, the interior scheme used is that of standard explicit second-order central differences in space and time; see Reference [23] for more details. In all the examples the computed solution is compared with a reference solution obtained numerically in a much larger domain, but with the same level of discretization. It is thought to be wise not to refine the spatial or temporal discretization for the reference solution, since what should be checked here is the effectivity of the boundary treatment while all other computational parameters are fixed. Analytical solutions exist for only very simple cases; see, e.g. the tests done in Reference [23].

\subsection{Quarter plane example}

In our first example we employ a quarter plane shown in Figure 2. The semi-infinite domain $\mathscr{D}$ is bounded by the physical boundaries $\Gamma_{\mathrm{S}}$ and $\Gamma_{\mathrm{W}}$. A large finite domain, denoted $\mathscr{D}^{*}$, where a reference solution $\eta_{\text {ref }}$ will be calculated, is represented by a $10 \times 10$ square with a $40 \times 40$ mesh. The truncated computational domain $\Omega$ is a $5 \times 5$ square with a $20 \times 20$ mesh and bounded by $\Gamma_{\mathrm{S}}, \Gamma_{\mathrm{W}}$ and the artificial boundaries $\Gamma_{\mathrm{N}}$, and $\Gamma_{\mathrm{E}}$. On both domains 


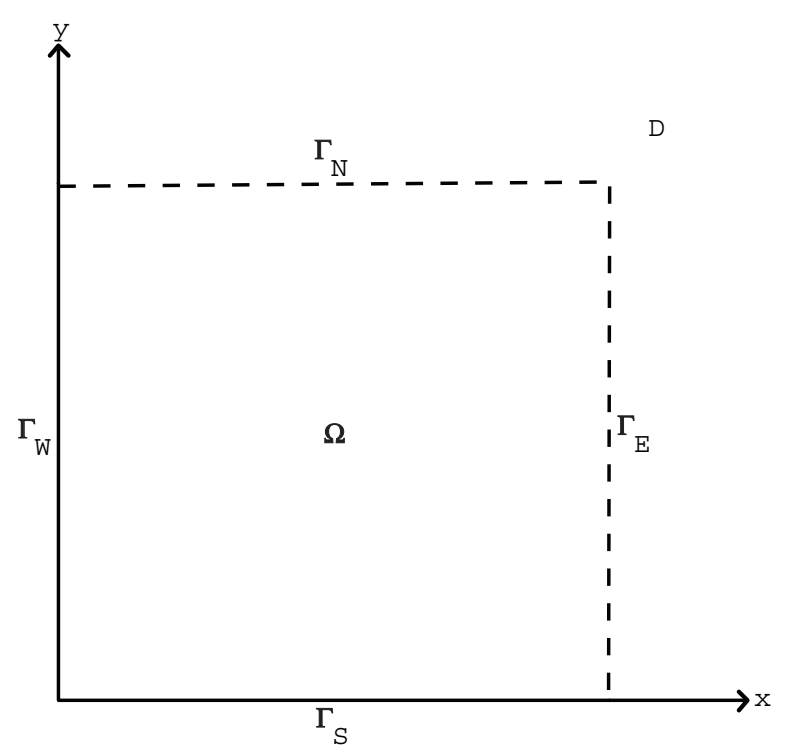

Figure 2. The semi-infinite quarter plane.

$\Delta x=0.25, \Delta y=0.25$, and $\Delta t=0.025$. A reference speed of $C_{0}=1$ and a dispersion parameter $f=0.5$ are used. The problem is run for 1000 time steps. To complete the problem description, a Neumann condition is imposed on $\Gamma_{\mathrm{S}}$, i.e.

$$
\frac{\partial \eta}{\partial y}=0
$$

and a Dirichlet condition is imposed on $\Gamma_{\mathrm{W}}$ and is given by

$$
\eta_{\mathrm{W}}(y, t)= \begin{cases}\sum_{m=1}^{3} A_{m} \cos \left(\frac{n_{m} \pi(y-1.875)}{3.75}\right) \sin \left(\omega_{m} t\right) & \text { if } y \leqslant 3.75 \\ 0 & \text { otherwise }\end{cases}
$$

where the parameters $A_{m}, n_{m}$, and $\omega_{m}$ are selected as follows:

$$
\begin{aligned}
A_{m} & =0.001,0.002,0.001 \\
n_{m} & =1,3,1 \\
\omega_{m} & =0.81,1.37,1.68
\end{aligned}
$$



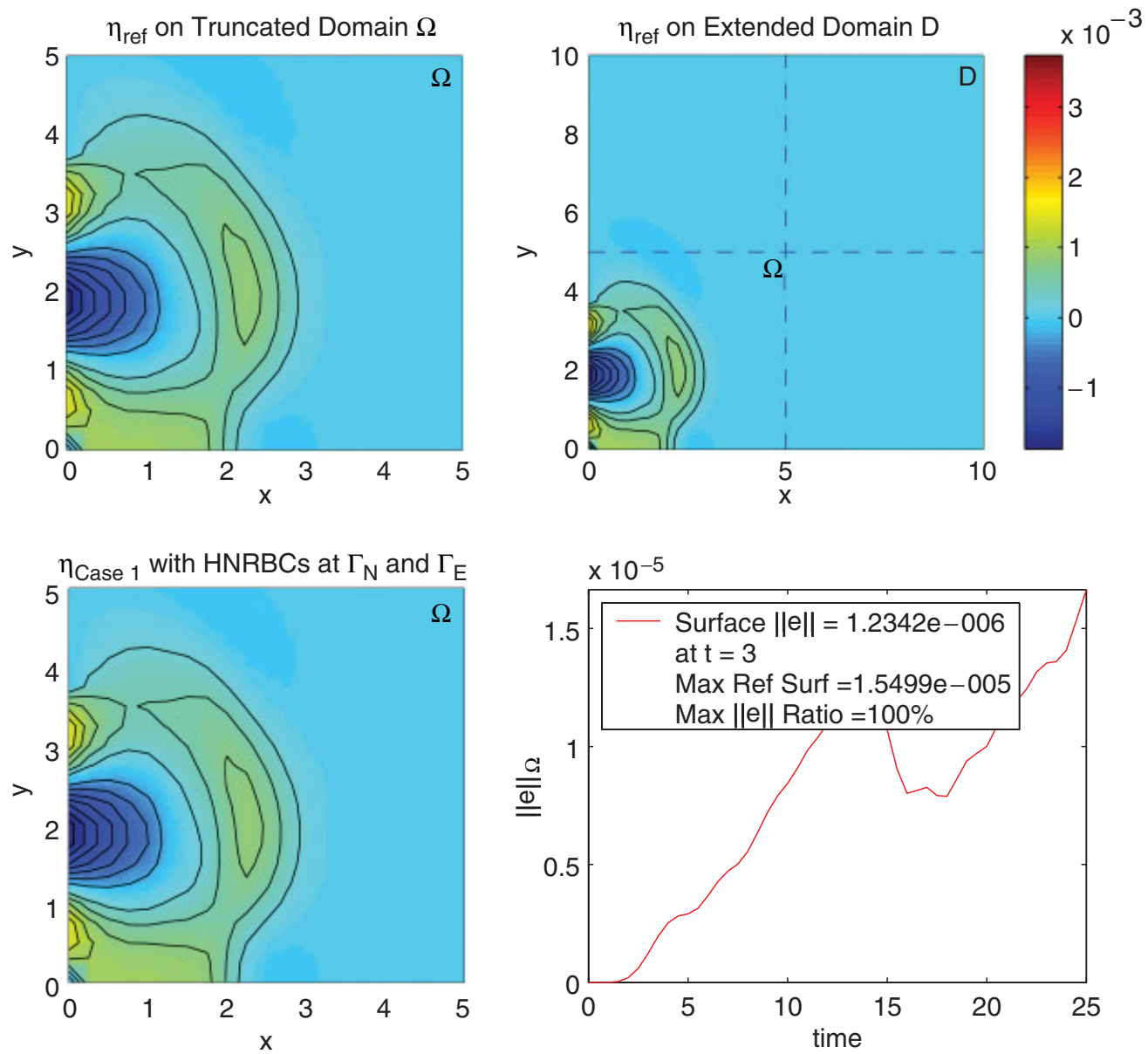

Figure 3. Wave pulse passes through $\Gamma_{\mathrm{N}}$ at $t=3$.

The Higdon NRBC of order $J=5$ is imposed on $\Gamma_{\mathrm{N}}$ and $\Gamma_{\mathrm{E}}$, with $C_{j}=1 / \sqrt{2}, j=1, \ldots, 5$ on both boundaries. The high-derivative implementation described in Section 3.1 is used. Since the same $C_{j}$ 's are used for the two boundaries, the parameters and operators used to discretize the $\Gamma_{\mathrm{E}}$ and $\Gamma_{\mathrm{N}}$ boundary conditions are identical.

A solution $\eta_{\text {ref }}$ and $\eta$ is computed for $\mathscr{D}^{*}$ and $\Omega$, respectively, using explicit second-order finite differencing. Filled contour plots are generated for each solution. In Figure 3 , these solutions are reported at $t=3$. The upper-right subplot displays the solution for $\eta_{\text {ref }}$ on $\mathscr{D}^{*}$. The upper-left subplot is a magnification of $\eta_{\text {ref }}$ on the sub-domain $\Omega$ and represents a solution on $\Omega$ as if no boundaries were present at $x=5$ and $y=5$. The lower-left subplot reports the solution for $\eta$ on $\Omega$. A qualitative comparison can be made between the two left-side subplots to determine the effectiveness of the NRBC in restricting the domain. A quantitative measurement of this comparison appears in the lower-right subplot, which reports an error measure $\|e(t)\|_{\Omega}$ 

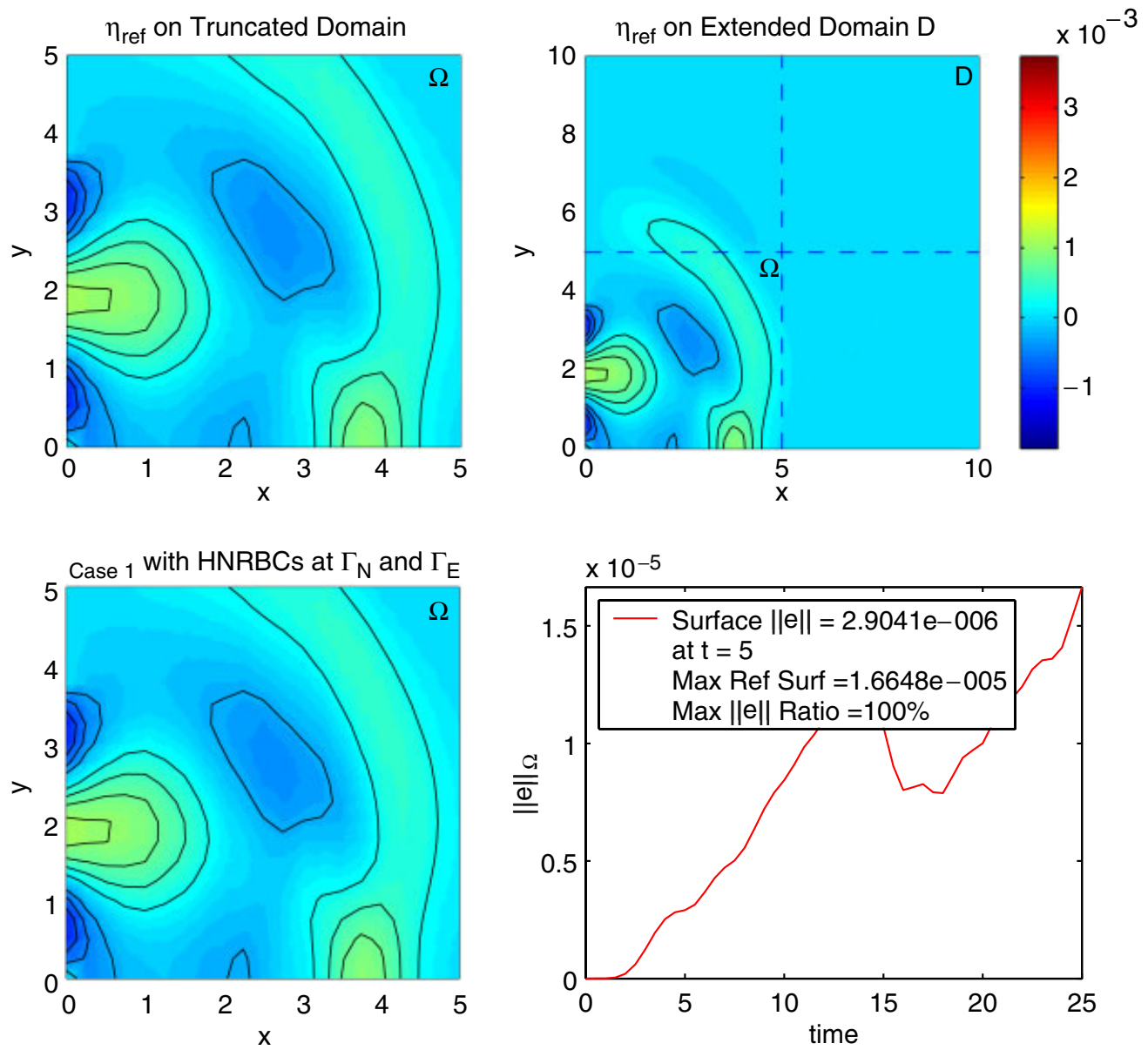

Figure 4. Wave pulse passes through $\Gamma_{\mathrm{E}}$ at $t=5$.

versus $t$. Here

$$
\|e(t)\|_{\Omega}=\sqrt{\sum_{i=1}^{N_{x}} \sum_{j=1}^{N_{y}} \frac{\left[\eta_{\mathrm{ref}}\left(x_{i}, y_{j}, t\right)-\eta\left(x_{i}, y_{j}, t\right)\right]^{2}}{N_{x} N_{y}}}
$$

and $N_{x}=N_{y}=20$ is the number of grid points in each direction. Note that for computational purposes, Higdon NRBCs with $C_{j}=\{1,1,1,1,1,1,1\}$ are imposed at $y=10$ and $x=10$ on $\mathscr{D}^{*}$. Any reflection from these extended boundaries of $\mathscr{D}^{*}$ will have little or no effect on the domain of interest $\Omega$. If we conduct the run for shorter times, such waves will not reach back into $\Omega$.

The almost linear increase in time seen in the lower right error plot (here and in all subsequent figures), has nothing to do with the boundary treatment, but is associated with the linear increase of error which is known to exist in all standard finite difference schemes for 

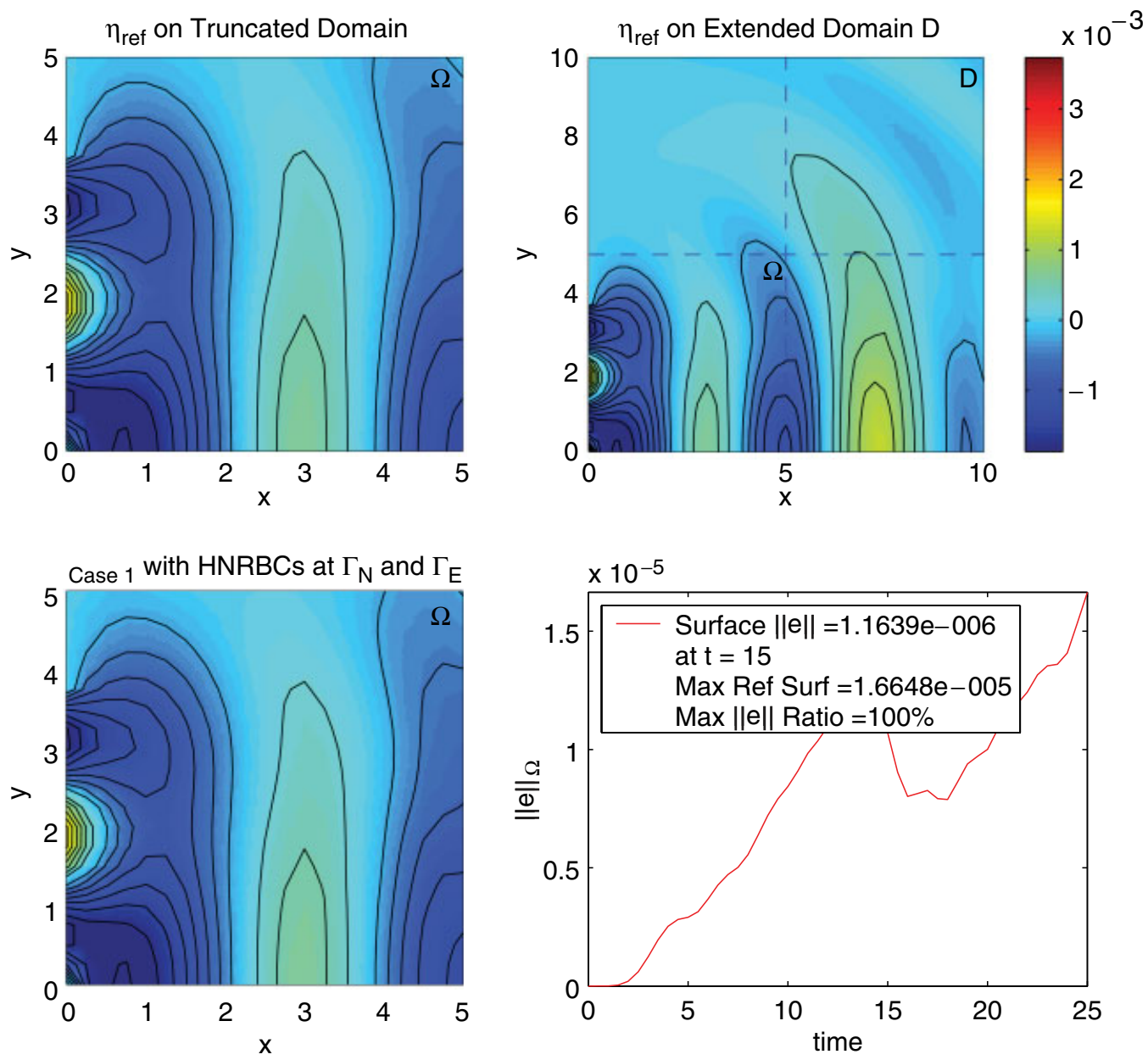

Figure 5. Wave pulse passes through corner at $t=15$.

hyperbolic problems that are not specially designed to control global dispersion ('pollution') errors.

In Figure 3 at $t=3$, the wave pulse has almost reached $\Gamma_{\mathrm{N}}$. The magnitude of the error measurement is less than the error due to the discretization. In Figure 4 at $t=5$, the wave pulse has reached $\Gamma_{\mathrm{N}}$ and passes without undue increase in $\|e(t)\|_{\Omega}$. Note that a leading front of the pulse is about to reach the intersection of $\Gamma_{\mathrm{N}}$ and $\Gamma_{\mathrm{E}}$. In Figure 5 at $t=15$, the wave has passed through the corner unimpeded with no adverse effect on $\|e(t)\|_{\Omega}$. The measured error $\|e(t)\|_{\Omega}$ at $t=25$ is shown in Figure 6. (Note the large errors from the corner in the reference solution; these however do not affect the scheme in $\Omega$.)

At the corner grid points the Higdon conditions were applied similarly to any other point, except that one-sided second-order FD approximations were employed for the tangential derivatives instead of central FDs. One should note, however, that two different ways to approximate 

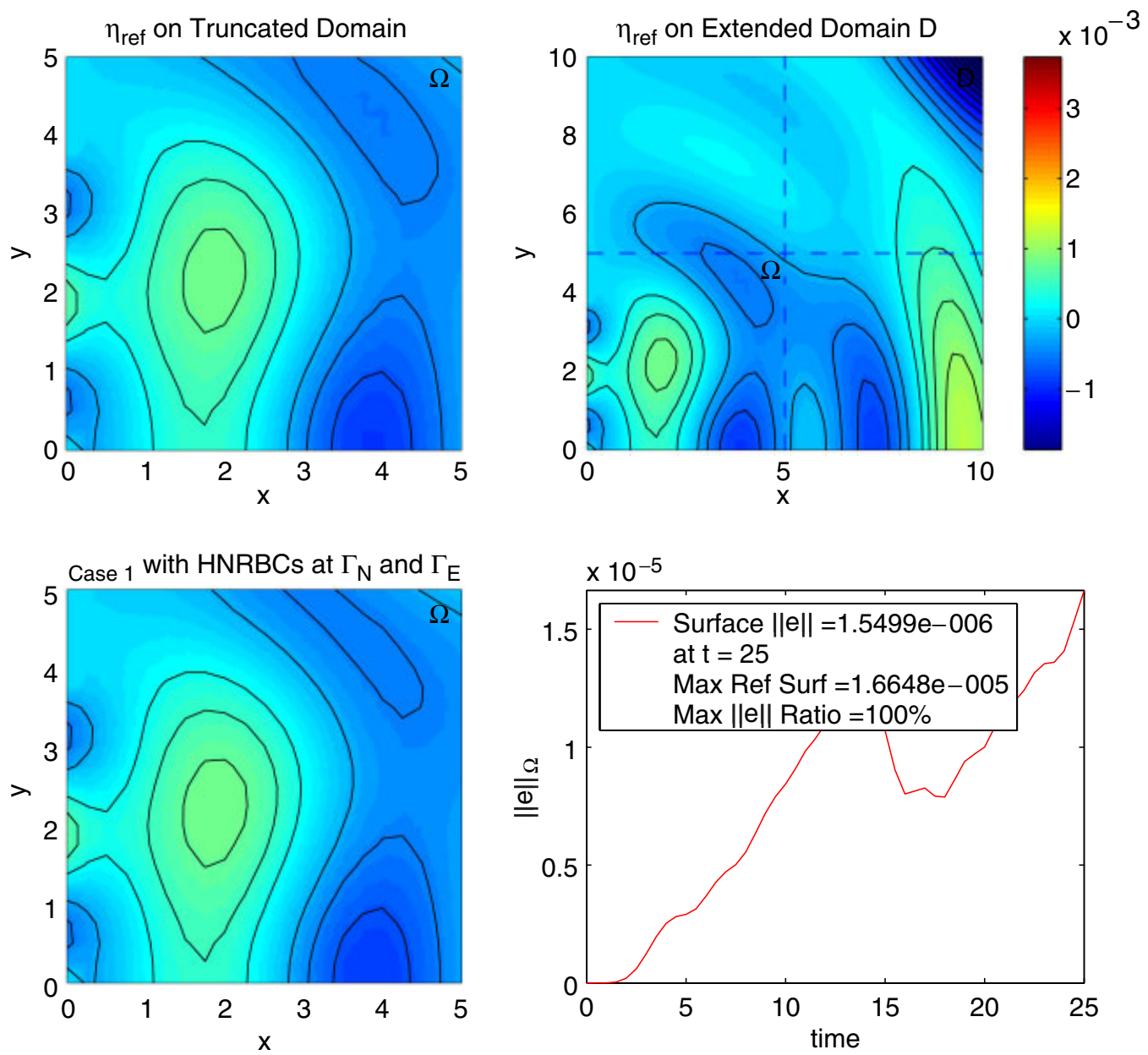

Figure 6. Large errors at the corner at $t=25$.

Table II. Error as function of $J$.

\begin{tabular}{lc}
\hline Order & Error \\
\hline 1 & $9.7362(-5)$ \\
2 & $2.376(-5)$ \\
3 & $5.9458(-6)$ \\
4 & $2.063(-6)$ \\
5 & $1.1795(-6)$ \\
\hline
\end{tabular}

the solution at the corner points are possible. For example, in the northeast corner one may evaluate the solution based on boundary values calculated for $\Gamma_{\mathrm{E}}$, or based on the $\Gamma_{\mathrm{N}}$ boundary values. Numerical experiments show that the two procedures yield the same results. 

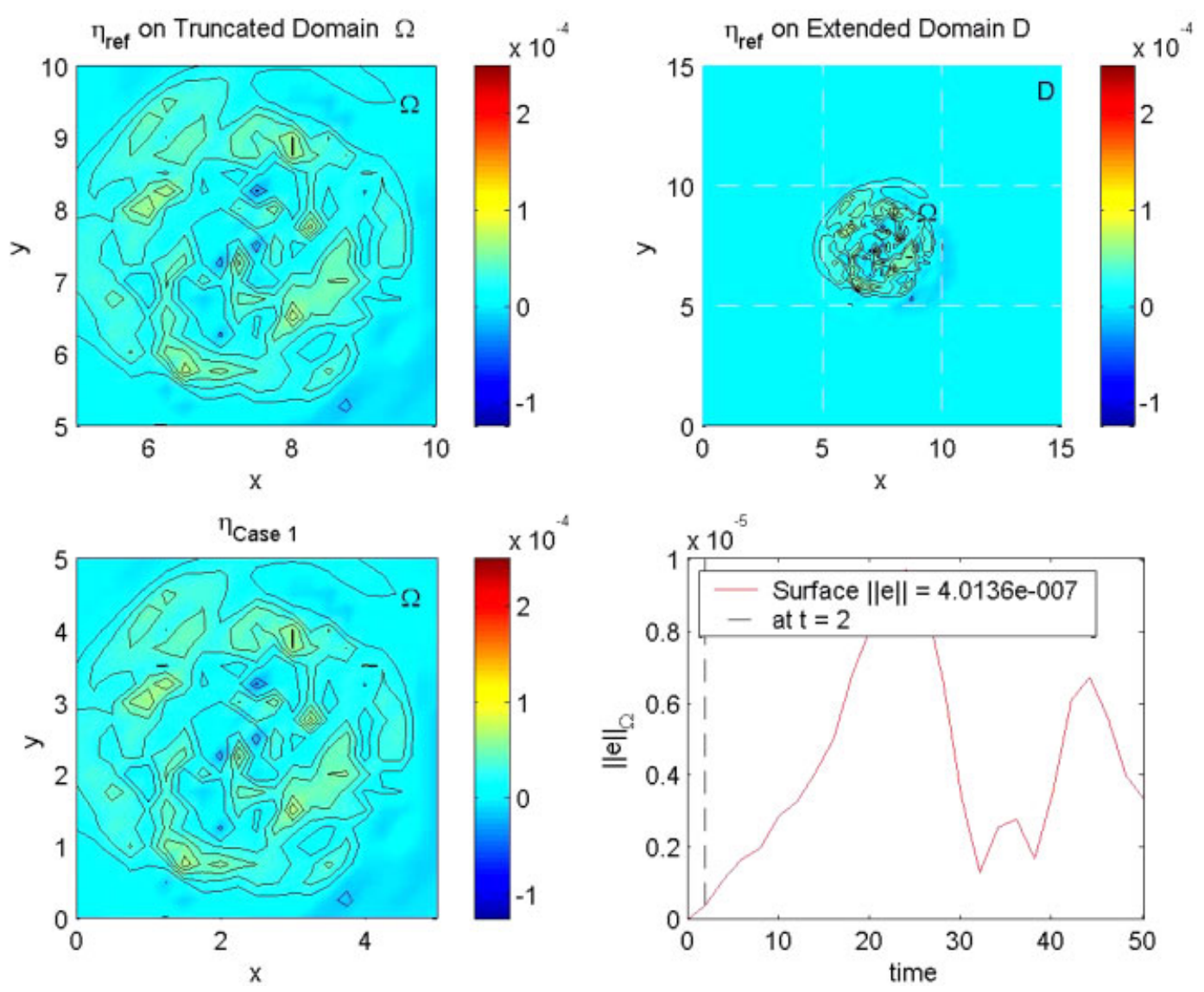

Figure 7. Solution at time $t=2$ after the surface disturbance given by (38) has been evolved.

\subsection{NRBC high order}

The next example involves the use of several values of the parameter $J$ to solve the same quarter plane problem. The only change is that for the reference solution we have used $J=5$ and all $C_{j}=1 / \sqrt{2}$ and for the inner domain we kept $C_{j}=1 / \sqrt{2}$ but allowed $J$ to vary from 1 to 5 . The integration time step is $\Delta t=0.025$ and the final time is $t=10$. The maximum error as a function of $J$ is given in Table II. It is clear that the error is smaller with increasing $J$.

A remark should be made regarding the reduction of error obtained by increasing the order $J$. Of course spatial and temporal discretization errors always exists 'in the background' and there is no point in increasing $J$ beyond the point where the boundary error due to the use of the $J$ th-order NRBC is smaller than the discretization error. This limit can be seen in actual computations if $J$ in increased gradually until the numerical results 'converge' to a result that does not change with further $J$ increase. Some estimate on this limit $J$ may be obtained from an error analysis of both the discretization error and the boundary error; such analysis is outside 

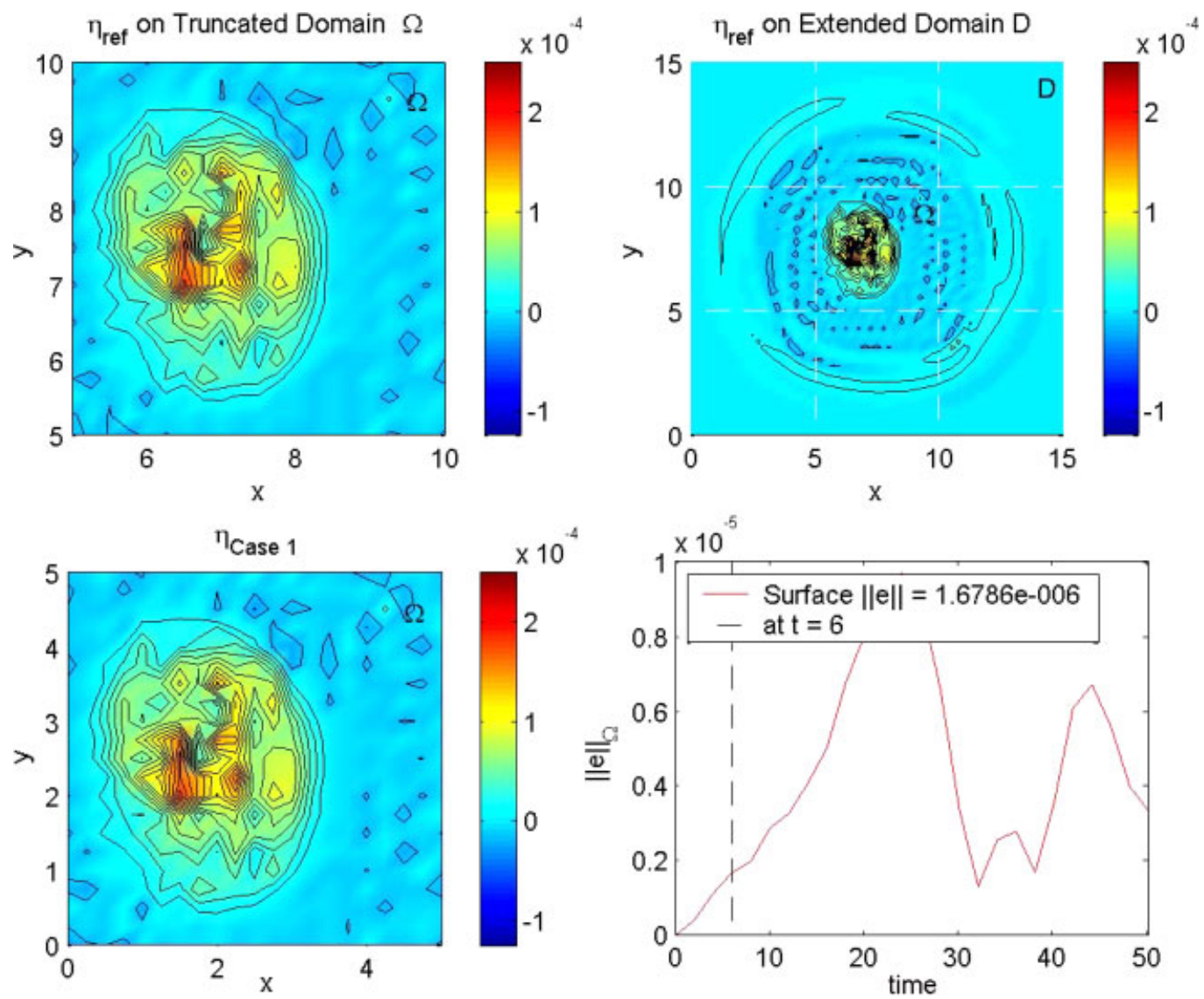

Figure 8. Solution at time $t=4$, surface disturbance has left $\Omega$ and another disturbance has been initiated.

the scope of this paper (and has not been done, to the best of our knowledge, by other authors in other cases of high-order NRBCs). Of course, the finer the discretization is, the higher $J$ can (and should) be made.

\subsection{Four sides}

In the next example we consider a truncated domain $\Omega$ where Higdon NRBCs are imposed on all four sides. The boundary conditions on $\Gamma_{\mathrm{N}}$ and $\Gamma_{\mathrm{E}}$ are discretized as before. The boundary conditions on $\Gamma_{\mathrm{S}}$ and $\Gamma_{\mathrm{W}}$ are discretized in a similar manner, however forward- vice backward-difference approximations are used for the spatial derivatives. As before, $\Omega$ is a $5 \times 5$ square with a $20 \times 20$ mesh. Higdon NRBCs are located at $x=0, y=0, x=5$, and $y=5$ with $C_{j}=\{1,1,1,1,1\}$ at each boundary.

The extended domain $\mathscr{D}$ is the infinite plane. The reference domain $\mathscr{D}^{*}$ is a $15 \times 15$ square with a $60 \times 60$ mesh. The domain of interest $\Omega$ is located in the centre of $\mathscr{D}^{*}$ at $5 \leqslant x, y \leqslant 10$. Higdon boundaries are placed at $x=0, y=0, x=15$, and $y=15$ with $C_{j}=\{1,1,1,1,1,1,1\}$ 

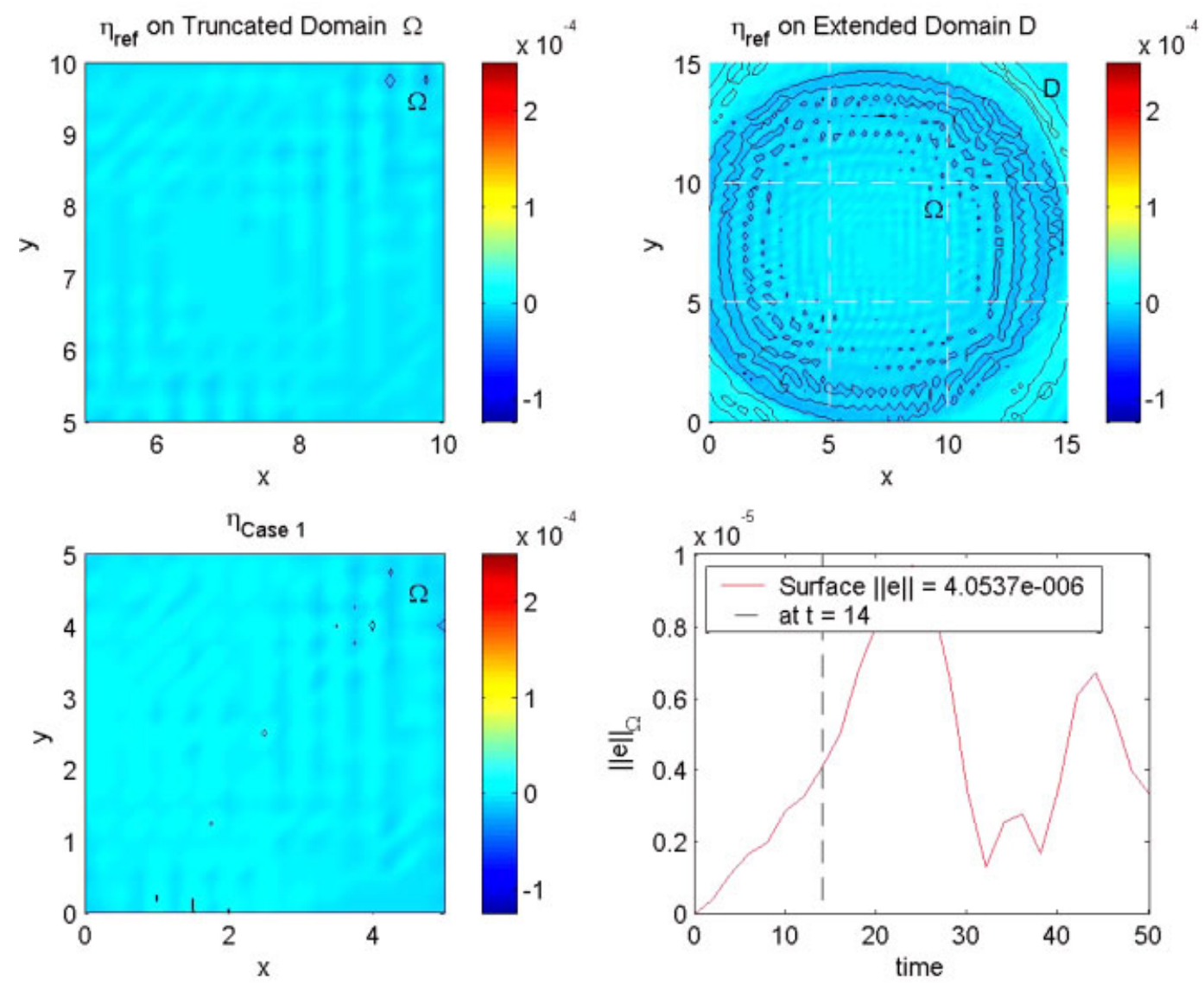

Figure 9. Solution at time $t=14$, both events have passed through the boundaries.

at each boundary. This is done for computational purposes only and any spurious reflection from these extended boundaries will not significantly pollute the domain of interest unless the integration time is long enough. On both domains $\Delta x=\Delta y=0.25$ and the time-step $\Delta t=0.1$. Again, $C_{0}=1$ and $f=0.5$.

As typically done in meteorological simulations, random values that represent physical disturbances or 'events' in $\Omega$, which are highly non-smooth and non-symmetric, are introduced at designated times at specified $x$ and $y$ ranges. At the given time, these random values are added to existing values of $\eta$. The dispersive mechanism defined by (3) is then allowed to act on the event. These random events are sufficiently complex in nature to expose any difficulty with the Higdon NRBCs.

For this example two separate events are imposed. Event 1 is a surface event given by

$$
S^{t=0.1}= \begin{cases}0.0001 * \operatorname{rand}(-0.5,0.5) & \text { if } 1.5 \leqslant x, y \leqslant 3.5 \\ 0 & \text { otherwise }\end{cases}
$$



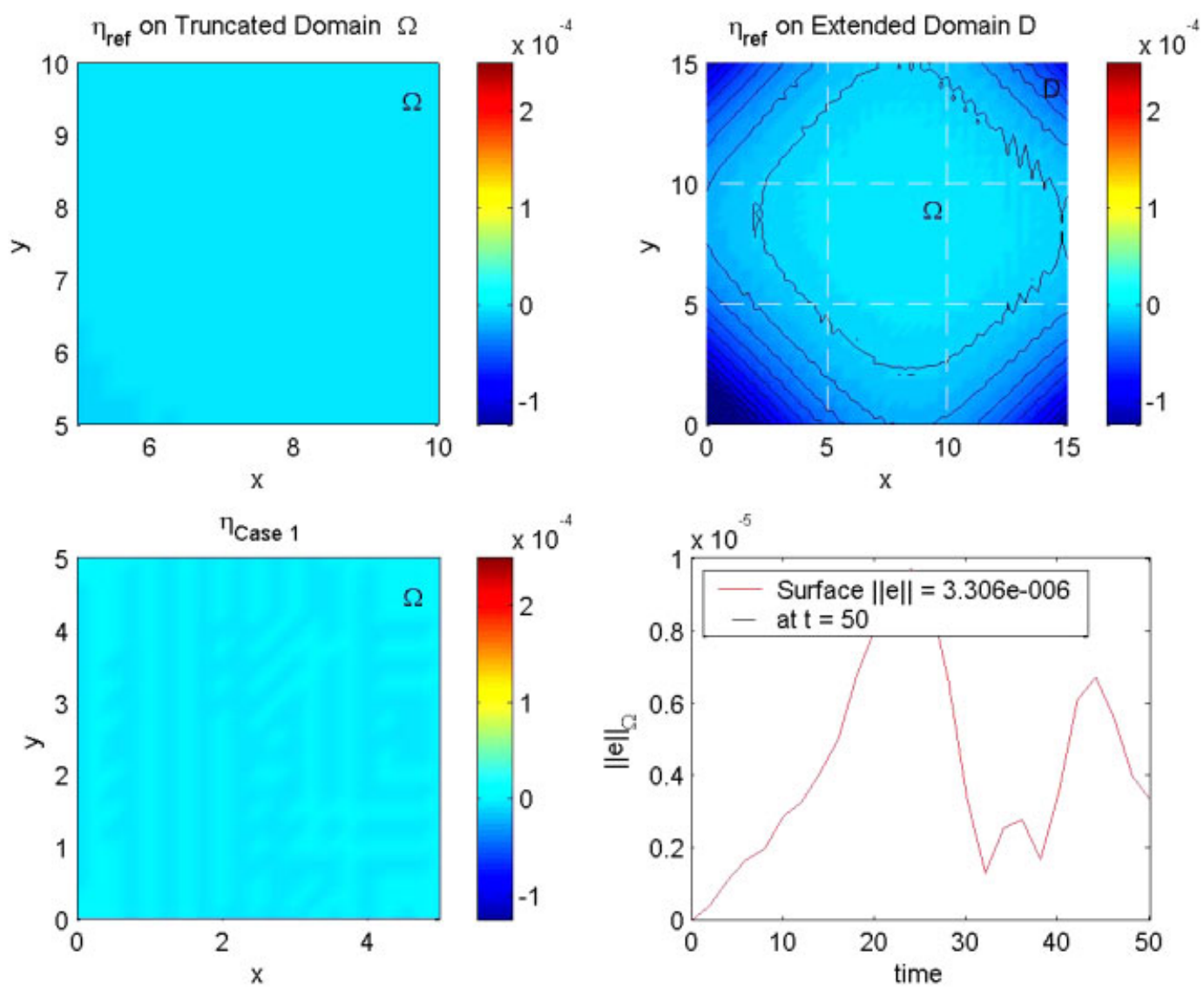

Figure 10. Solution at $t=50$, long after events have passed through the boundaries.

where $S^{t=0.1}(x, y)$ represents a disturbance initiated at $t=0.1$ and $\operatorname{rand}(-0.5,0.5)$ is a random number on the interval $[-0.5,0.5]$. Event 2 at $t=5$ is given by

$$
S^{t=5}= \begin{cases}0.00015 * \operatorname{rand}(-0.25,0.75) & \text { if } 1.5 \leqslant x \leqslant 2.25 \text { and } 1.5 \leqslant y \leqslant 3.5, \\ 0 & \text { otherwise }\end{cases}
$$

All events must be shifted 5 units in the $x$ and $y$ directions when initiating activity on $\mathscr{D}^{*}$, in order to properly place them in the domain's centre.

A trial is run for 50 time units. In Figure 7 at $t=2$, the surface event has been initiated and the resulting waves are propagating toward the four sides of $\Omega$. The error measurement $\|e(t)\|_{\Omega}$ is near zero even though the wave already crossed the boundary of $\Omega$. In Figure 8 at $t=4$, the first event has left $\Omega$ with no significant spurious reflection at either the boundaries or the corners. In Figure 9 at $t=14$, both events have passed through the artificial boundary successfully. An outward radiating wave front can be seen in the upper-right subplot of the extended domain $\mathscr{D}^{*}$. The left-side subplots are remarkably similar indicating that Higdon boundary 

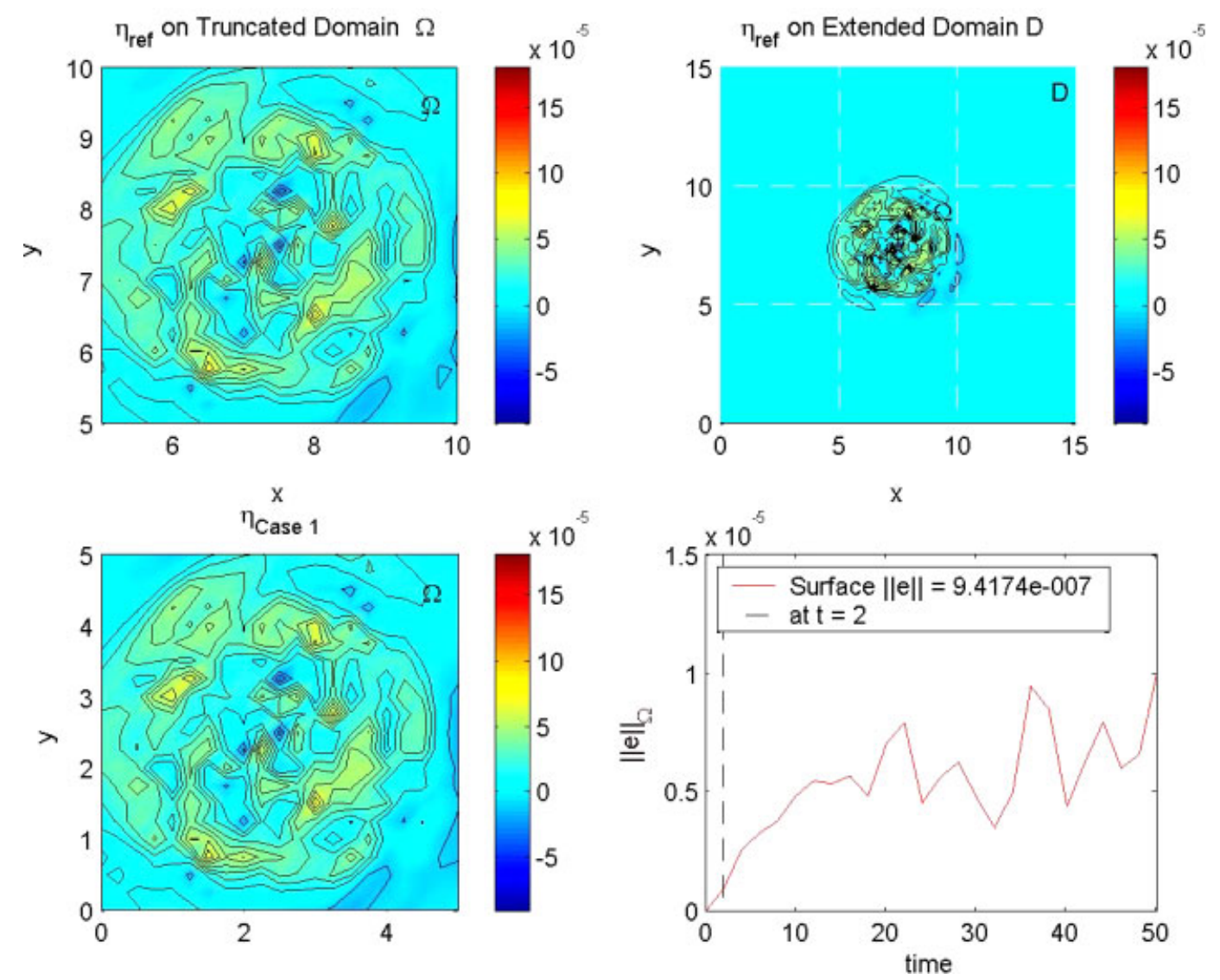

Figure 11. Auxiliary variable formulation. Solution at $t=2$ after the surface disturbance given by (38) has been evolved.

conditions are suitable. In Figure 10 at $t=50$, most of the wave energy resulting from the disturbances has left $\Omega$. The effect due to spurious reflection starts to become significant since the solution is nearly zero, thus increasing the error measurement for $\Omega$. (This effect, which often occurs in wave problems, may be controlled by using more sophisticated albeit more complicated and less 'physical' error measures.)

\subsection{Auxiliary variables}

In our last example we use the auxiliary variable formulation discussed in Section 3.2 with $J=7$ and $C_{j}=\{1,1,1,1,1,1,1\}$. All other parameters are unchanged, except that fresh random values are chosen for the two wave events. In Figure 11 at $t=2$ the surface event has been initiated. This figure is similar to the previous example. Figure 12 shows the solution at $t=4$. As before, we can see that the wave crosses the boundary without reflection. The error norm is slightly larger but it is still very small. In Figure 13 we show the solution at $t=14$, after the wave had passed through the outer boundary of the larger domain $\mathscr{D}^{*}$. Again the performance of 

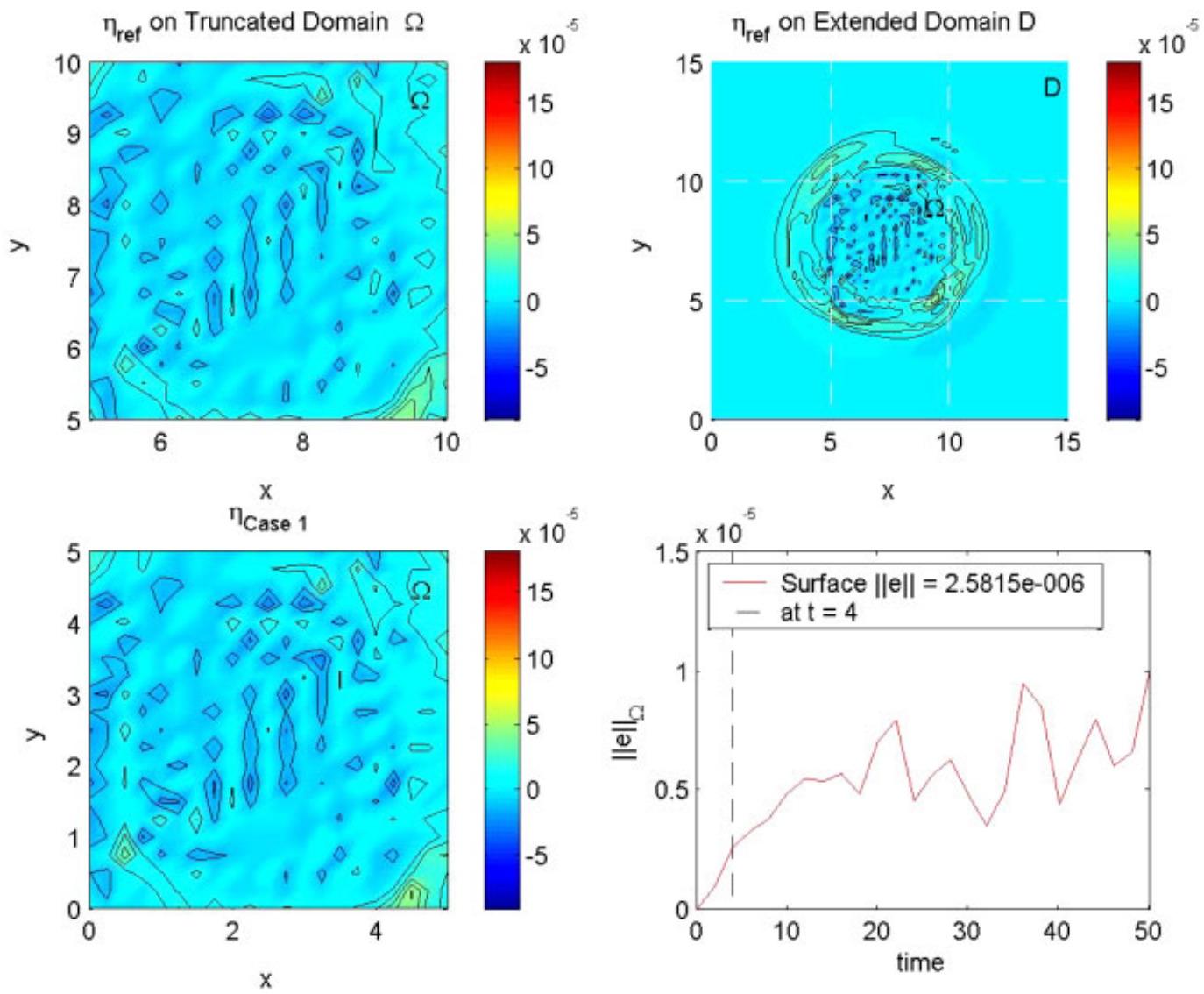

Figure 12. Auxiliary variable formulation. Solution at $t=4$ after the first surface disturbance has left $\Omega$ and another disturbance initiated.

the scheme is excellent. We have also present the solution after long integration. At $t=50$ the solution is given in Figure 14. Notice that the error is a magnitude larger than the corresponding one in the previous example.

Compared to the formulation of Collino [10], in which the NRBC has the form of a onedimensional wave equation on the boundary, in our auxiliary-variable formulation the solution near the corners (during the duration of the current experiment) seems to be smooth and accurate even without any special corner treatment. This is probably due to the special form of (32) with $\alpha_{j}=0$ (which is the case due to $C_{j}=C_{0}$ ), namely the fact the only a first-order time derivative appears in the NRBC. Nevertheless, for long times and high $J$ 's corner instability does occur, as shown below.

As mentioned above, the high-derivative formulation yielded stable results in all the experiments performed. This is so regardless of the computation time duration, the order $J$ or the grid density. On the other hand, the auxiliary-variable formulation exhibited long-time instability, probably due to the lack of compatibility corner conditions. We have observed from additional 

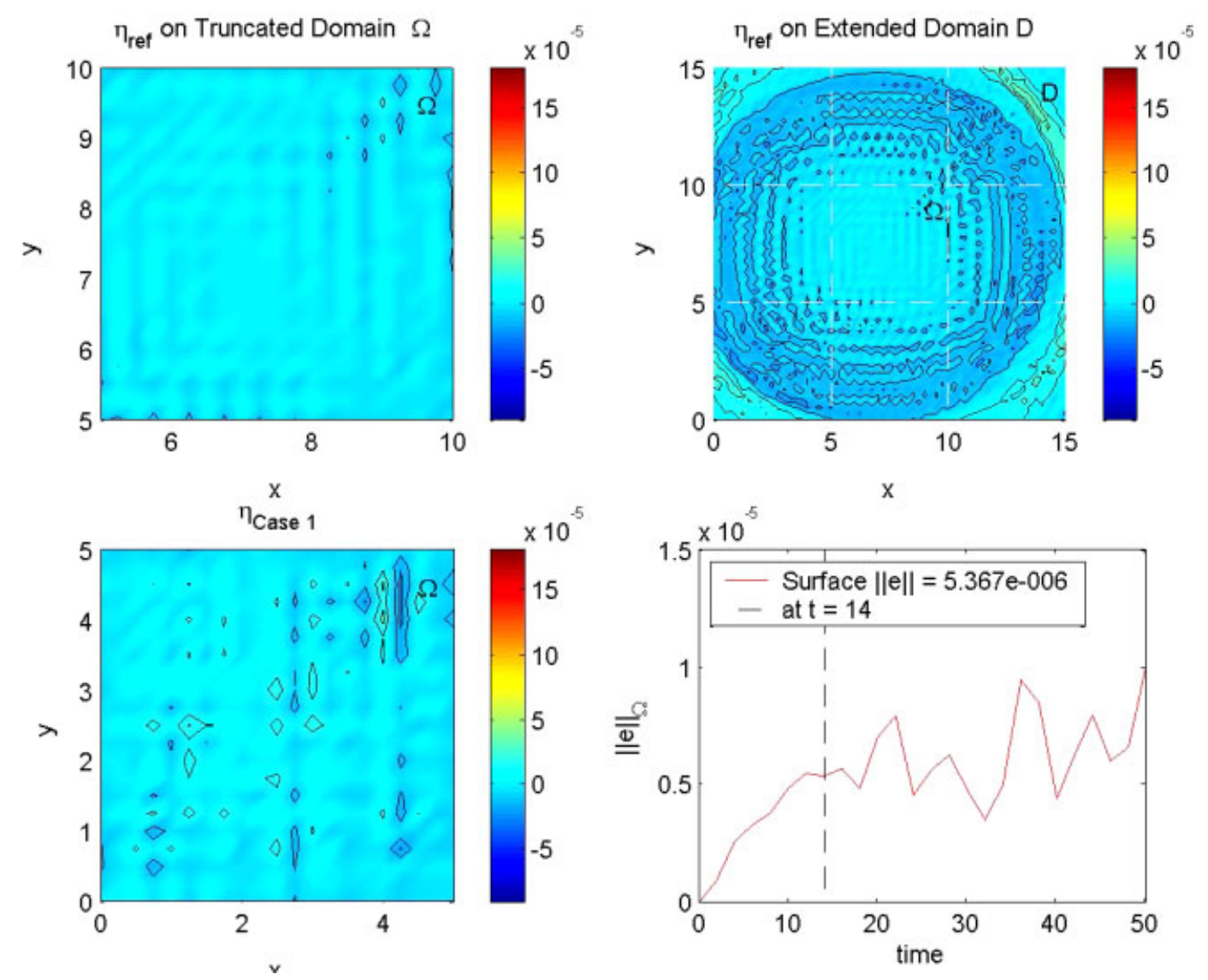

Figure 13. Auxiliary variable formulation. Solution at $t=14$ after both events have passed through the boundaries.

numerical experiments not presented here that for higher orders $J$ the instability appears earlier. Also, for a fixed $J$, refining the grid has the effect of delaying the instability (in addition to improving the accuracy as long as the solution is stable).

Construction of appropriate compatibility conditions for the corners in the auxiliary-variable formulation is a major work in itself, and is left for the future.

\section{CONCLUSIONS}

We considered here two reformulations of the Higdon NRBCs: one that involves high-order derivatives with a special discretization scheme, and another that does not involve any high derivatives beyond second order. The latter formulation is made possible by introducing special auxiliary variables on the boundary. 

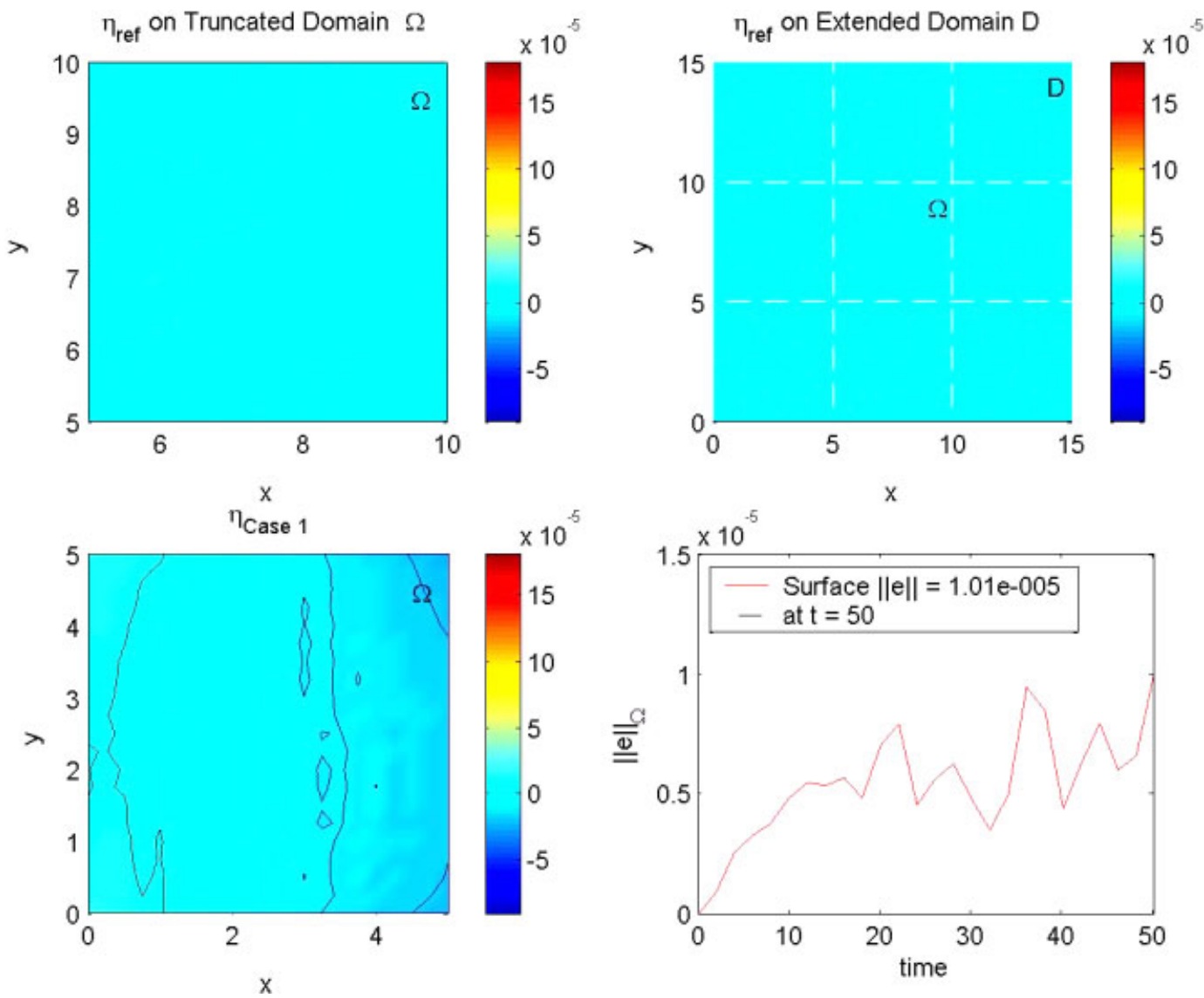

Figure 14. Auxiliary variable formulation. Solution at $t=50$ long after both events have passed through the boundaries.

Both formulations were incorporated here in a finite difference scheme. The auxiliary variable formulation can also be incorporated in a finite element scheme. This has been done in Reference [31] for the case of a wave guide, and is yet to be done for the exterior problem. Extending our previous papers with similar formulations, here the method was applied to a fully exterior two-dimensional problem with a rectangular boundary.

In the high-derivative scheme the order can be as high as the thickness of the 'buffer zone', whereas in the auxiliary-variable scheme it can be arbitrarily high, provided that no corner instability occurs. Long-time instability does occur in the auxiliary-variable formulation (but not in the high-derivative formulation) unless the corners are treated specially. The corner problem should be addressed, for example along the lines of Reference [35]; however it is beyond the scope of the present paper.

Obviously the choice of scheme and the value of the order $J$ are a tradeoff of a few factors, the main ones being the accuracy desired and computing time. The additional computing time associated with the NRBC is proportional to $J$ in the auxiliary-variable formulation and 
exponentially growing with $J$ in the high-derivative formulation. Of course, there is no point in using an unnecessarily large $J$; the largest $J$ that should be used is the value for which the NRBC error is of the same order of magnitude as the basic discretization error (in space and time). A prudent choice of $J$ should be made adaptively, based on some a posteriori error analysis. Moreover, the value of $J$ may vary in different locations on $\mathscr{B}$ and in different time steps.

In light of all the factors outlined above, it is felt that once the corner-stability issue in the auxuliary-variable formulation is resolved, this formulation is going to be superior over the highderivative formulation. The latter can be thought of as 'brute-force' high-order differentiator in time and space, while the former is a more elegant and general way to circumvent the need for high derivatives. Therefore, no attempt has been made in this paper to perform an actual numerical comparison between the two schemes for various cases.

Related future work will include investigation of the issue of corner stability, as explained above, and the adaptation of the proposed approach to more complicated configurations, such as three-dimensional problems in the presence of wave dispersion. The latter configuration is only technically, not essentially, more complicated than the two-dimensional one dealt with here. Also, the high-order NRBCs will be applied to the Shallow Water Equations, which serve as an important testbed for more complicated dispersive models in meteorology. In addition, an attempt to adapt the new NRBCs to curved artificial boundaries will be made via variable transformation, although in certain applications (like meteorology) geometries other than rectangular are not regarded as relevant.

See further discussion in the thesis of the first author, [47].

\section{ACKNOWLEDGEMENTS}

This work was supported in part by the Office of Naval Research (ONR), and the Naval Postgraduate School (NPS).

\section{REFERENCES}

1. Givoli D. Numerical Methods for Problems in Infinite Domains. Elsevier: Amsterdam, 1992.

2. Zienkiewicz OC, Newton RE. Coupled vibrations of a structure submerged in a compressible fluid. Proceedings of the Interernational Symposium on Finite Element Techniques, Stuttgart, 1969; 359-379.

3. Lysmer J, Kuhlemeyer RL. Finite dynamic model for infinite media. Journal of Engineering and Mechanical Division (ASCE) 1969; 95:859-877.

4. Smith WD. A nonreflecting plane boundary for wave propagation problems. Journal of Computational Physics 1974; 15:492-503.

5. Givoli D. Non-reflecting boundary conditions: a review. Journal of Computational Physics 1991; 94:1-29.

6. Tsynkov SV. Numerical solution of problems on unbounded domains, a review. Applied Numerical Mathematics 1998; 27:465-532.

7. Givoli D. Exact representations on artificial interfaces and applications in mechanics. Applied Mechanics Review 1999; 52:333-349.

8. Hagstrom T. Radiation boundary conditions for the numerical simulation of waves. Acta Numerica 1999; 8:47-106

9. Bayliss A. Turkel E. Radiation boundary conditions for wave-like equations. Communications on Pure and Applied Mathematics 1980; 33:707-725.

10. Collino F. High order absorbing boundary conditions for wave propagation models. Straight line boundary and corner cases. In Proceedings of the Second International Conference on Mathematical and Numerical Aspects of Wave Propagation, Kleinman R et al. (eds). SIAM: Delaware, 1993; 161-171. 
11. Grote MJ, Keller JB. Exact nonreflecting boundary conditions for the time dependent wave equation. SIAM Journal on Applied Mathematics 1995; 55:280-297.

12. Grote MJ, Keller JB. Nonreflecting boundary conditions for time dependent scattering. Journal of Computational Physics 1996; 127:52-65.

13. Grote MJ, Keller JB. Nonreflecting boundary conditions for elastic waves. SIAM Journal on Applied Mathematics 2000; 60:803-819.

14. Sofronov IL. Conditions for complete transparency on the sphere for the three-dimensional wave equation. Russian Academy of Sciences Doklacly Mathematics 1993; 46:397-401.

15. Sofronov IL. Artificial boundary conditions of absolute transparency for two- and three-dimensional external time-dependent scattering problems. European Journal of Applied Mathematics 1998; 9:561-588.

16. Hagstrom T, Hariharan SI. Progressive wave expansions and open boundary problems. In Computational Wave Propagation, Engquist B, Kriegsmann GA (eds), IMA Volumes in Mathematics and its Applications, vol. 86. Springer: New York, 1997; 23-43.

17. Hagstrom T, Hariharan SI. A formulation of asymptotic and exact boundary conditions using local operators. Applied Numerical Mathematics 1998; 27:403-416.

18. Guddati MN, Tassoulas JL. Continued-fraction absorbing boundary conditions for the wave equation. Journal of Computational Acoustics 2000; 8:139-156.

19. Givoli D. High-order non-reflecting boundary conditions without high-order derivatives. Journal of Computational Physics 2001; 170:849-870.

20. Givoli D, Patlashenko I. An optimal high-order non-reflecting finite element scheme for wave scattering problems. International Journal for Numerical Methods in Engineering 2002; 53:2389-2411.

21. Pedlosky J. Geophysical Fluid Dynamics. Springer: New York, 1987.

22. Navon IM, Neta B, Hussaini MY. A perfectly matched layer approach to the linearized shallow water equations models. Monthly Weather Review 2004; 132:1369-1378.

23. Givoli D, Neta B. High-order non-reflecting boundary conditions for dispersive waves. Wave Motion 2003; 37:257-271.

24. Givoli D, Neta B. High-order non-reflecting boundary scheme for time-dependent waves. Journal of Computational Physics 2003; 186:24-46.

25. Higdon RL. Radiation boundary conditions for dispersive waves. SIAM Journal on Numerical Analysis 1994; 31:64-100.

26. Higdon RL. Absorbing boundary conditions for difference approximations to the multi-dimensional wave equation. Mathematics of Computation 1986; 47:437-459.

27. Higdon RL. Numerical absorbing boundary conditions for the wave equation. Mathematics of Computation 1987; 49:65-90.

28. Higdon RL. Radiation boundary conditions for elastic wave propagation. SIAM Journal on Numerical Analysis 1990; 27:831-870.

29. Higdon RL. Absorbing boundary conditions for elastic waves. Geophysics 1991; 56:231-241.

30. Higdon RL. Absorbing boundary conditions for acoustic and elastic waves in stratified media. Journal of Computational Physics 1992; 101:386-418.

31. Givoli D, Neta B, Igor Patlashenko. Finite element solution of exterior time-dependent wave problems with high-order boundary treatment. International Journal for Numerical Methods in Engineering 2003; 58:1955-1983.

32. Ramahi OM. Stability of absorbing boundary conditions. IEEE Transactions on Antennas and Propagation 1999; 47:593-599.

33. Bamberger A, Joly P, Roberts JE. Second-order absorbing boundary conditions for the wave equation: a solution for the corner problem. SIAM Journal on Numerical Analysis 1990; 27:323-352.

34. Vacus O. Mathematical analysis of absorbing boundary conditions for the wave equation: the corner problem. Mathematics of Computation 2005; 74:177-200.

35. Hagstrom T, Warburton T. A new auxiliary variable formulation of high-order local radiation boundary conditions: corner compatibility conditions and extensions to first order systems. Wave Motion 2004; 39: 327-338.

36. Ditkowski A, Gottlieb D. On the Engquist-Majda absorbing boundary conditions for hyperbolic systems. In AMS Contemporary Mathematics, vol. 330, Recent Advances in Scientific Computing and PDEs, 2003.

37. Pearson RA. Consistent boundary conditions for the numerical models of systems that admit dispersive waves. Journal of Atmospheric Science 1974; 31:1418-1489. 
38. Orlanski I. A simple boundary condition for unbounded hyperbolic flows. Journal of Computational Physics 1976; 21:251-269.

39. Raymond WH, Kuo HL. A radiation boundary condition for multi-dimensional flows. Quarterly Journal of the Royal Meteorological Society 1984; 110:535-551.

40. Miller MJ, Thorpe AJ. Radiation conditions for the lateral boundaries of limited-area numerical models. Quarterly Journal of the Royal Meteorological Society 1981; 107:615-628.

41. Klemp JB, Lilly DK. Numerical simulation of hydrostatic mountain waves. Journal of Atmospheric Science 1978; 35:78-107.

42. Wurtele M, Paegle J, Sielecki A. The use of open boundary conditions with the storm-surge equations. Monthly Weather Review 1971; 99:537-544.

43. Hodur RM. The naval research laboratory's coupled ocean/atmosphere mesoscale prediction system (COAMPS). Monthly Weather Review 1997; 125:1414-1430.

44. Ren X, Wang KH, Jin KR. Open boundary conditions for obliquely propagating nonlinear shallow-water waves in a wave channel. Computers and Fluids 1997; 26:269-278.

45. Jensen TG. Open boundary conditions in stratified ocean models. Journal of Marine Systems 1998; 16: 297-322.

46. Oliveira FSBF. Improvement on open boundaries on a time dependent numerical model of wave propagation in the nearshore region. Ocean Engineering 2000; 28:95-115.

47. van Joolen VJ. Application of Higdon non-reflecting boundary conditions to shallow water models. Ph.D. Thesis, Naval Postgraduate School, Monterey, CA, 2003. 